TRANSACTIONS OF THE

AMERICAN MATHEMATICAL SOCIETY

Volume 353, Number 6, Pages 2347-2370

S 0002-9947(00)02625-8

Article electronically published on October 11, 2000

\title{
RELATIVE EMBEDDING PROBLEMS
}

\author{
ELENA V. BLACK AND JOHN R. SWALLOW
}

\begin{abstract}
We consider Galois embedding problems $G \rightarrow H \cong \operatorname{Gal}(X / Z)$ such that a Galois embedding problem $G \rightarrow \operatorname{Gal}(Y / Z)$ is solvable, where $Y / Z$ is a Galois subextension of $X / Z$. For such embedding problems with abelian kernel, we prove a reduction theorem, first in the general case of commutative $k$-algebras, then in the more specialized field case. We demonstrate with examples of dihedral embedding problems that the reduced embedding problem is frequently of smaller order. We then apply these results to the theory of obstructions to central embedding problems, considering a notion of quotients of central embedding problems, and classify the infinite towers of metacyclic $p$-groups to which the reduction theorem applies.
\end{abstract}

\section{INTRODUCTION}

A classical question of Galois theory is the embedding problem: whether a given Galois extension may be embedded into a larger Galois extension with specified group. Embedding problems, considered variously over number fields and geometric fields, offer approaches both to the inverse Galois problem and to the determination of number-theoretic consequences implied by a particular Galois group. When solving embedding problems, it is often productive to determine relationships between the embedding problem at hand and related embedding problems, so that both the solvability conditions and the field solutions, if any, of one embedding problem may be used to advantage in the other.

In this paper we follow such an approach, considering embedding problems with the additional information that a related embedding problem has a solution. Given a Galois extension $E_{B} / K$ with Galois group $H$ and a surjection of groups $G \rightarrow H$, we ask if $E_{B} / K$ embeds into a $G$-Galois extension of $K$, with the added knowledge that some Galois subextension of $E_{B}$, say $K_{1} / K$ with Galois group $\bar{H}$, embeds in a Galois extension over $K$ with Galois group $G$. We call these relative embedding problems and study such problems in detail when the kernel of the surjection $G \rightarrow \bar{H}$ is abelian. Our results on relative embedding problems, culminating in Theorem 3.1 in the context of fields, reduce the question of solvability of certain embedding problems with abelian kernel to the question of solvability of reduced embedding problems. These reduced embedding problems are of smaller order when the centralizers of the abelian kernels are larger than the kernels themselves.

Received by the editors January 4, 1999 and, in revised form, August 20, 1999.

2000 Mathematics Subject Classification. Primary 12F12, 13B05; Secondary 12F10.

The first author gratefully acknowledges a University of Oklahoma Junior Faculty Research Grant. The second author gratefully acknowledges support under National Science Foundation Grant No. DMS-9501366 and a Davidson College MacArthur Faculty Study and Research Grant. 
The primary motivation for our work on this problem is derived from the case of dihedral groups of 2-power order. (For a survey of work on similar embedding problems see [GSS]; for later work, see $[\mathrm{Cr}$ and [Le].) Suppose that $K$ is a field and that $G=D_{2^{d}}$ is a dihedral group of order $2^{d+1}$. The group $G=D_{2^{d}}$ naturally appears in a tower $\left\{G_{i}=D_{2^{i}}\right\}$ of central $C_{2^{-} \text {-extensions }}$

$$
G=G_{d} \longrightarrow G_{d-1} \longrightarrow \cdots \longrightarrow G_{1}=C_{2} \times C_{2} \longrightarrow G_{0}=C_{2}
$$

Suppose further that $M_{1} / K$ is a $C_{2} \times C_{2}$-Galois extension which has a solvable embedding problem for $G$, with solution $M_{d} / K$. Let $M_{0} / K$ be the fixed field in $M_{d}$ of the $C_{2^{d}}$-subgroup of $G$. Now for $2 \leq i \leq d$, consider $M_{i} / K$, the intermediate $D_{2^{i}}$-Galois subextension of $M_{d} / K$. Each field $M_{i}$ is obtained by adjoining a square root of some element of $M_{i-1}^{\times}$, so that $M_{i}=M_{i-1}\left(\sqrt{\gamma_{i-1}}\right)$, where $\gamma_{i-1} \in M_{i-1}^{\times}$. It is well-known (see [BI, Lemma 4.1], for instance) that the other embeddings of $M_{i-1} / K$ into $G_{i}$-Galois extensions differ only by a $K^{\times}$-constant $r$, i.e., these other embeddings are given by fields $M_{i-1}\left(\sqrt{r \gamma_{i-1}}\right)$. Using our Main Theorem we determine for which $r$ the extensions $M_{i-1}\left(\sqrt{r \gamma_{i-1}}\right) / K$ embed in Galois extensions with group $G$ as well. We show in Theorem 3.3 that $M_{i-1}\left(\sqrt{r \gamma_{i-1}}\right) / K$ embeds in a Galois extension with group $G$ if and only if the extension $M_{0}(\sqrt{r}) / K$ embeds in a Galois extension with group $G_{d-i+1}$ cyclic over $M_{0}$.

In section 1 we introduce the machinery of the relative embedding problem and Baer products of group extensions and Galois extensions. We then develop in section 2 correspondences among relative embedding problems and related split and reduced embedding problems. In section 3 we present the main reduction theorem, with an immediate application to dihedral relative embedding problems. In section 4 we present implications for the structure of obstructions of central embedding problems, and in section 5 we determine towers, particularly infinite towers, of metacyclic $p$-group extensions to which our reduction theorem applies.

We are grateful to the host institution, the Mathematisches Forschungsinstitut Oberwolfach, and the organizers, D. Harbater, B. H. Matzat and Y. Ihara, of the conference "Galois Groups and Fundamental Groups," for a discussion which became the starting point for this paper. We also thank the Department of Mathematics at the University of Oklahoma for its hospitality provided to the second author during some of the collaboration.

\section{Notation, Definitions, And Background}

Let $k$ be a field. Unless stated otherwise, throughout this paper a $k$-algebra denotes a commutative $k$-algebra, and an extension of algebras $S / R$, or an algebra extension, denotes an extension of commutative $k$-algebras. Below we introduce embedding problems and relative embedding problems in this context. For definitions and basic results in the Galois theory of commutative rings, see [CHR] or [DI]. It is important to note that, in contrast with the theory over fields, a Galois extension of $k$-algebras $S / R$ does not uniquely determine the Galois group and its action; to make precise a Galois extension, one must specify the extension $S / R$ of $k$-algebras, the group $G$, and an explicit isomorphism $\nu: G \stackrel{\simeq}{\longrightarrow} \operatorname{Aut}(S / R)$ such that the subring of $S$ fixed by $\nu(G)$ is $R$. Sometimes this extension is denoted by the pair $(S / R, \nu)$ and is called a $G$-Galois extension (of algebras). Each of the following definitions subsumes a corresponding definition in the Galois theory of fields. 
Definition 1.1. Let $f: G \rightarrow H$ be a surjection of finite groups with kernel $B$, and let $\left(S_{B} / R, \nu\right)$ be an $H$-Galois extension. The embedding problem associated to $\left(S_{B} / R, \nu, f\right)$ is to determine if there exists a $G$-Galois extension $(S / R, \eta)$ such that $S_{B} \subset S$ and $h \eta=\nu f$, where $h$ is the homomorphism $h: \eta(G) \rightarrow \nu(H)$ with kernel $\eta(B)$ of Galois theory:

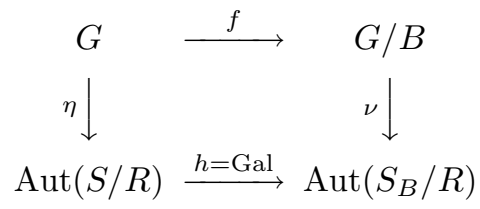

We call an embedding problem abelian, central, or Frattini if $B$ is abelian, $B$ lies in the center $Z(G)$ of $G$, or $B$ lies in the Frattini subgroup $\Phi(G)$ of $G$, respectively.

If the embedding problem associated to $\left(S_{B} / R, \nu, f\right)$ has an affirmative answer for an Galois extension $S_{B} / R$, we say that $S_{B} / R$ has solvable embedding problem for $\nu$ and $f$. If the context is clear, we may omit $\nu$ and/or $f$ and write that $S_{B} / R$ has solvable embedding problem for $G$.

Definition 1.2. Suppose that we have two embedding problems, one associated to $S_{1} / R, \eta_{1}: H_{1} \stackrel{\simeq}{\longrightarrow} \operatorname{Aut}\left(S_{1} / R\right)$, and $f_{1}: G_{1} \rightarrow H_{1}$, and one associated to $S_{2} / R$, $\eta_{2}: H_{2} \stackrel{\simeq}{\longrightarrow} \operatorname{Aut}\left(S_{2} / R\right)$, and $f_{2}: G_{2} \rightarrow H_{2}$. We say that the embedding problems are common over a Galois extension $R_{1} / R$ if $R_{1}$ lies in the intersection of $S_{1}$ and $S_{2}$. By Galois theory, then, there exist normal subgroups $A_{1} \subset G_{1}$ and $A_{2} \subset G_{2}$ such that $S_{1}^{\eta_{1}\left(A_{1}\right)}=S_{2}^{\eta_{2}\left(A_{2}\right)}=R_{1}$ and $G_{1} / A_{1} \cong G_{2} / A_{2}$.

In the sequel the notation $\operatorname{Fix}(X)$ will denote the subgroup of the Galois group of the extension under consideration which fixes the subalgebra $X$.

Definition 1.3. A relative embedding problem consists of an embedding problem associated to $S_{B} / R, \nu: H \stackrel{\simeq}{\longrightarrow} \operatorname{Aut}\left(S_{B} / R\right)$, and $f: G \rightarrow H$; a Galois subalgebra extension $R_{1} / R$ with quotient action $\nu^{\prime}: H / \operatorname{Fix}\left(R_{1}\right) \stackrel{\simeq}{\longrightarrow} \operatorname{Aut}\left(R_{1} / R\right)$; and a solution $(T / R, \eta)$ to an embedding problem with group $G$ associated to $\left(R_{1} / R, \nu^{\prime}\right)$ and the quotient surjection $f^{\prime}: G \rightarrow H / \operatorname{Fix}\left(R_{1}\right)$, such that under $\eta$ the subgroup in $G$ corresponding to $R_{1}$ contains ker $f$.

Informally, a relative embedding problem is a pair of embedding problems with group $G$, common over a Galois subalgebra extension $R_{1} / R$, such that one embedding problem is solvable. A relative embedding problem is then an embedding problem with additional information. The following commutative diagram helps connect the various embedding problems contained in the definition of a relative embedding problem. Let $B=\operatorname{ker} f$, so that $H=G / B$ and the subgroup of $H$ corresponding to $R_{1} / R$ is $A / B$. 


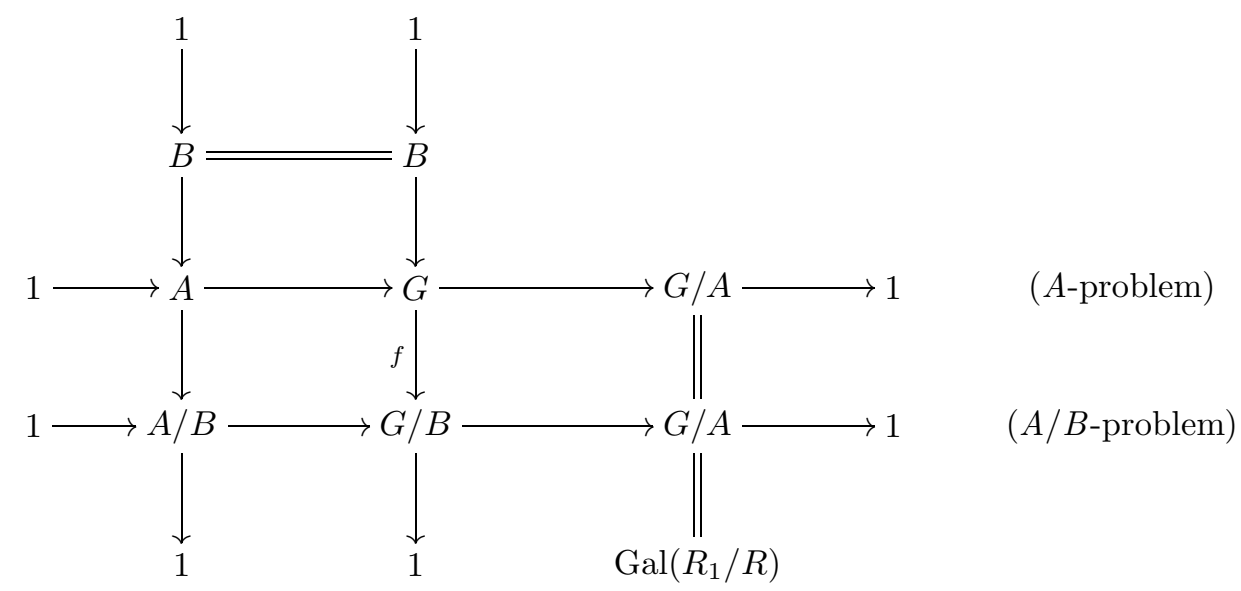

(B-problem)

Considered in this fashion, we are given in the definition of a relative embedding problem a solution $\left(S_{B} / R, \nu\right)$ of the $A / B$-embedding problem and a solution $(T / R, \eta)$ of the $A$-embedding problem, and we seek a solution of the $B$-embedding problem associated to the $G / B$-Galois extension $\left(S_{B} / R, \nu\right)$.

Under the further hypothesis that $R_{1} / R$ corresponds to an abelian subgroup of $G$, i.e., the subgroup $A$ in the above diagram is abelian, we now define the principal object of study in this paper, the class of abelian relative embedding problems.

Definition 1.4. An abelian relative embedding problem is an embedding problem associated to $S_{B} / R, \nu: H \stackrel{\simeq}{\longrightarrow} \operatorname{Aut}\left(S_{B} / R\right)$, and $f: G \rightarrow H$; a Galois subalgebra extension $R_{1} / R$ with quotient action $\nu^{\prime}: H / \operatorname{Fix}\left(R_{1}\right) \stackrel{\simeq}{\longrightarrow} \operatorname{Aut}\left(R_{1} / R\right)$; and a solution $(T / R, \eta)$ to an embedding problem with group $G$ associated to $\left(R_{1} / R, \nu^{\prime}\right)$ and the quotient surjection $f^{\prime}: G \rightarrow H / \operatorname{Fix}\left(R_{1}\right)$, such that under $\eta$ the subgroup in $G$ corresponding to $R_{1}$ is abelian and contains ker $f$. It follows that $\operatorname{ker} f$ is abelian and hence the embedding problem associated to $S_{B} / R$ is abelian.

We note that several results of [ILF §3.15] can be viewed as a treatment of abelian relative embedding problems in the case $S_{B}=R_{1}$.

We now recall from [LF] definitions of Baer products of extensions (of groups and of Galois extensions).

Definition 1.5. Let $B$ be an abelian group. Let $G_{1}$ and $G_{2}$ be finite groups which are group extensions of a finite group $H$ by a $H$-module $B$; hence we have exact sequences $1 \rightarrow B \rightarrow G_{1} \stackrel{\phi_{1}}{\longrightarrow} H \rightarrow 1$ and $1 \rightarrow B \rightarrow G_{2} \stackrel{\phi_{2}}{\longrightarrow} H \rightarrow 1$. Let $\tilde{G}$ be the product of $G_{1}$ and $G_{2}$ with amalgamated quotient group $H$, consisting of elements $\left(g_{1}, g_{2}\right)$ such that $\phi_{1}\left(g_{1}\right)=\phi_{2}\left(g_{2}\right)$. Identify $B$ with its image in each of $G_{1}$ and $G_{2}$. There is a natural surjection $\tilde{G} \rightarrow H$ with kernel $B \times B$, consisting of elements $\left(b_{1}, b_{2}\right), b_{1} \in B \subset G_{1}, b_{2} \in B \subset G_{2}$. Let $\tilde{B}$ consist of the elements $\left(b_{1}, b_{2}\right)$ where $b_{1}=b_{2}^{-1}$ under the identification of $B$. Then the Baer product (of groups) of $G_{1}$ and $G_{2}$ is the extension $\tilde{G} / \tilde{B}$, and we have an exact sequence

$$
1 \longrightarrow B \longrightarrow \tilde{G} / \tilde{B} \longrightarrow H \longrightarrow 1,
$$


where $B$ is embedded in $\tilde{G} / \tilde{B}$ by means of the map $b \mapsto(b, 1)(\bmod \tilde{B})$.

Note that the Baer product of groups depends not only on the subgroups $B$ in $G_{1}$ and $G_{2}$, but also on the particular injections of the groups $B$ into the corresponding subgroups in $G_{1}$ and $G_{2}$.

Recall the notion of equivalence of group extensions of a group $H$ by a $H$-module $B$. Let

$$
1 \longrightarrow B \stackrel{i_{1}}{\longrightarrow} G_{1} \stackrel{\phi_{1}}{\longrightarrow} H \longrightarrow 1
$$

and

$$
1 \longrightarrow B \stackrel{i_{2}}{\longrightarrow} G_{2} \stackrel{\phi_{2}}{\longrightarrow} H \longrightarrow 1
$$

describe two group extensions $G_{1}, G_{2}$ of $H$ by a $H$-module $B$. Then $\left(G_{1}, i_{1}, \phi_{1}\right)$ and $\left(G_{2}, i_{2}, \phi_{2}\right)$ are defined to be equivalent if there exists an isomorphism $\theta: G_{1} \stackrel{\simeq}{\longrightarrow} G_{2}$ such that $\theta i_{1}=i_{2} \operatorname{id}_{B}$ and $\phi_{2} \theta=\operatorname{id}_{H} \phi_{1}$. The Baer product is then a composition law on the classes of equivalent group extensions, under which these classes form an abelian group isomorphic to $H^{2}(H, B)$ ILF, Theorem 3.15.1]. Recall that the inverse class of $G$, an arbitrary extension of $H$ by $B$, is given as follows. Define the group $\bar{G}$ as a set of elements $\bar{g}, g \in G$, with the multiplication $\overline{g_{1}} \cdot \overline{g_{2}}=\overline{g_{2} g_{1}}$. Under the map $g \mapsto \bar{g}^{-1}, G$ is abstractly isomorphic to $\bar{G}$. The group $\bar{G}$ is an extension of $H$ by $B$, under the same action on $B$ as before, and the Baer product of $G$ and $\bar{G}$ is therefore the semidirect extension of $H$ by $B$. Note that the map $g \mapsto \bar{g}^{-1}$ is the automorphism of $B$ given by inversion.

Definition 1.6 ([LF §3.15]). Let $G_{1}$ and $G_{2}$ be finite groups which are group extensions of a finite group $H$ by a $H$-module $B$, where the injections of $B$ into $G_{1}$ and $G_{2}$ are fixed. Let $\left(S_{1} / R, \eta_{1}\right)$ be a $G_{1}$-Galois extension and $\left(S_{2} / R, \eta_{2}\right)$ be a $G_{2^{-}}$ Galois extension. Suppose that $S_{1} / R$ and $S_{2} / R$ share a subextension $R_{1} / R$ which is the fixed subalgebra of $B_{1} \subset G_{1}$ in $S_{1}$ and $B_{2} \subset G_{2}$ in $S_{2}$. Identify $B_{1}$ and $B_{2}$ with $B$ through the injections of $B$. Then let $\tilde{G} / \tilde{B}$ be the Baer product of groups $G_{1}$ and $G_{2}$ as defined above, and let $\tilde{S}=S_{1} \otimes_{R_{1}} S_{2}$ with action $\eta_{3}: \tilde{G} \rightarrow \operatorname{Aut}(\tilde{S} / R)$ defined by $\left(\eta_{3}\left(g_{1}, g_{2}\right)\right)\left(s_{1} \otimes s_{2}\right)=\eta_{1}\left(g_{1}\right)\left(s_{1}\right) \otimes \eta_{2}\left(g_{2}\right)\left(s_{2}\right)$. For $\left(g_{1}, g_{2}\right) \in \tilde{G}$, we have $\nu_{1}\left(g_{1}\right)=\nu_{2}\left(g_{2}\right)$ when restricted to $R_{1}$, hence $\eta_{3}$ is well-defined. We emphasize this action on the tensor product by writing $\eta_{1} \otimes \eta_{2}$ in place of $\eta_{3}$.

The Baer product (of Galois extensions) of $\left(S_{1} / R, \eta_{1}\right)$ and $\left(S_{2} / R, \eta_{2}\right)$ is the subalgebra $U$ of $\tilde{S}$ fixed by $\tilde{B} \subset \tilde{G}$, with the quotient action derived from $\eta_{1} \otimes \eta_{2}$ under the factorization. The (Baer) inverse of a $G$-extension $(S / R, \nu)$ is the $\bar{G}$ extension $(S / R, \nu i)$, where $i: \bar{G} \rightarrow G$ is the isomorphism from the (Baer group) inverse of $G$ to $G$.

One immediately anticipates that the Baer product of Galois extensions gives a composition law on equivalence classes of Galois extensions $(S / R, \eta)$ which extend a given $H$-Galois extension $\left(S_{B} / R, \nu\right)$ by $B$-extensions $S / S_{B}$, where $B$ is a fixed $H$ module. While there is a standard notion of equivalence of group extensions (given above), there are several choices for equivalence of Galois extensions. Observe that when a Galois extension $(S / R, \eta)$ is given, an implicit choice of a lifting of each element $h \in H$ has been made to an element $g_{h} \in G$ such that $\eta\left(g_{h}\right)=\nu(h)$ on $S_{B}$; these choices, in turn, determine a 2-cocycle $z \in Z^{2}(H, B)$. We do not want to distinguish between actions $\eta$ which amount to no more than a different choice of liftings to the same automorphism group of $S / R$, which would correspond in the group setting to two elements of $Z^{2}(H, B)$ in the same class of $H^{2}(H, B)$. 
Hence we wish to consider two Galois extensions $\left(S_{1} / R, G_{1}, \eta_{1}\right),\left(S_{2} / R, G_{2}, \eta_{2}\right)$ which extend a given $H$-Galois extension $\left(S_{B} / R, \nu\right)$ by $B$-extensions, where $B$ is a fixed $H$-module, (Galois extension-) equivalent if there exists an $S_{B}$-isomorphism of algebras $\theta: S_{1} \rightarrow S_{2}$ such that, if an appropriate choice of lifts $\eta_{1}\left(g_{1, h}\right), \eta_{2}\left(g_{2, h}\right)$ of $\nu(h)$ are made into $\eta_{1}\left(G_{1}\right)$ and $\eta_{2}\left(G_{2}\right)$, then $\theta\left(s^{\eta_{1}\left(g_{1, h}\right) \eta_{1}(b)}\right)=\theta(s)^{\eta_{2}\left(g_{2, h}\right) \eta_{2}(b)}$ for all $s \in S, h \in H, b \in B$. Such an equivalence implies that the map $\gamma: G_{1} \rightarrow G_{2}$ given by $\gamma\left(g_{1, h} b\right)=g_{2, h} b$ satisfies $\theta\left(s^{\eta_{1}\left(g_{1}\right)}\right)=\theta(s)^{\eta_{2}\left(\gamma\left(g_{1}\right)\right)}$ for $g_{1} \in G$. The group law in $G_{1}$ is

$$
\left(g_{1, h_{1}} b_{1}\right)\left(g_{1, h_{2}} b_{2}\right)=g_{1, h_{1}} g_{1, h_{2}} b_{1}^{h_{2}} b_{2},
$$

and since the analogous group law holds in $G_{2}$, the fact that

$$
\gamma\left(g_{1, h_{1}} g_{1, h_{2}} b_{1}^{h_{2}} b_{2}\right)=g_{1, h_{1}}^{\prime} g_{1, h_{2}}^{\prime} b_{1}^{h_{2}} b_{2}
$$

implies that $\gamma$ is an isomorphism. Abstractly, then, $G$ and $G^{\prime}$ are identical groups, and $\gamma$ leaves fixed the specified injections of $B$ into each group. We encapsulate this discussion in the following definition.

Definition 1.7 (ILF $\S 3.15 .2]$ ). Let $f: G \rightarrow H$ be a surjection of finite groups and let $\left(S_{B} / R, \nu\right)$ be an $H$-Galois extension. Two solutions $\left(S_{1} / R, \eta_{1}\right)$, and $\left(S_{2} / R, \eta_{2}\right)$ of the embedding problem associated to $\left(S_{B} / R, \nu, f\right)$ are said to be equivalent, or, following [ILF], equivalent in the broad sense, if there exist an $S_{B}$-isomorphism $\theta: S_{1} \rightarrow S_{2}$ and an automorphism $\gamma: G \rightarrow G$ trivial on $B=\operatorname{ker} f$ such that for all $s \in S_{1}, g \in G$,

$$
\theta\left(s^{\eta_{1}(g)}\right)=\theta(s)^{\left(\eta_{2} \gamma\right)(g)} .
$$

Remark 1.8. If $S_{1}, S_{2}$, and hence $S_{B}$ and $R$, are fields, then $S_{1}$ and $S_{2}$ are equivalent in the broad sense if and only if $S_{1}=S_{2}$ and $\eta_{1}^{-1} \eta_{2}$ is an automorphism of $G$ trivial on $B$ and identical on cosets $G / B$. Since $\theta$ is an $S_{B}$-isomorphism, the equivalence condition becomes $t^{\eta_{1}(g)}=t^{\left(\eta_{2} \gamma\right)(g)}$ for $t \in S_{B}$. Because $h \eta_{1}=h \eta_{2}=\nu f$, where $h$ is the surjection $\operatorname{Gal}\left(S_{i} / R\right) \rightarrow \operatorname{Gal}\left(S_{B} / R\right)$, then we have $t^{(\nu f)(g)}=t^{(\nu f \gamma)(g)}$ for $t \in S_{B}$; hence $f \gamma=f$, or $\gamma$ is identical on cosets $G / \operatorname{ker} f$.

Remark 1.9. The fact that the injection of $B$ into $G$ must be specified in the definitions of equivalence in the broad sense and the Baer product of Galois extensions raises the question of whether or not these definitions are unnecessarily restricted, i.e., whether or not two Galois extensions $\left(S / R, \eta_{1}\right)$ and $\left(S / R, \eta_{2}\right)$, extending $\left(S_{B} / R, \nu\right)$, isomorphic under an $S_{B}$-algebra isomorphism $\theta: S \rightarrow S$ and a group automorphism $\gamma: G \rightarrow G$ not trivial on the injections of $B$, satisfying $\theta\left(s^{\eta_{1}(g)}\right)=\theta(s)^{\eta_{2}(\gamma(g))}$, are also isomorphic under $\theta^{\prime}$ and $\gamma^{\prime}$, where $\gamma^{\prime}$ is trivial on $B$. When $S$ is a field, this situation cannot occur: any $S_{B}$-automorphism $\theta^{\prime}: S \rightarrow S$ is necessarily an element of the Galois group $\operatorname{Gal}\left(S / S_{B}\right)$, hence in $B$. Then the inner automorphism associated to $\theta^{\prime}$ leaves $B$ invariant.

When $S$ is not a field, however, it is possible to find such elements $\left(\theta^{\prime}, \gamma^{\prime}\right)$. Consider, for instance, the group $G=B=\langle\sigma\rangle \cong C_{4}$ and the completely splitting algebra $(S, \eta)$, where $S=\bigoplus_{\sigma^{i} \in B} R e_{\sigma^{i}}$ and $\eta\left(\sigma^{i}\right)\left(e_{b}\right)=e_{b \sigma^{i}}$ for $b \in B$, and set $S_{B}=R$ so that $H=1$. Define an $R$-automorphism $\theta: S \rightarrow S$ by $\theta\left(e_{\sigma^{i}}\right)=e_{\sigma^{-i}}$ and an automorphism $\gamma: B \rightarrow B$ by $\gamma(\sigma)=\sigma^{-1}$. Then $\gamma$ is not trivial on $B$. Write 


$$
\begin{aligned}
s=\sum_{i=0}^{3} r_{i} e_{\sigma^{i}} \text { for } s & \in S \text {. Then } \\
\theta\left(s^{\eta(b)}\right) & =\theta\left(\sum_{i=0}^{3} r_{i} e_{\sigma^{i}}^{\eta(b)}\right)=\theta\left(\sum_{i=0}^{3} r_{i} e_{\sigma^{i} b}\right)=\sum_{i=0}^{3} r_{i} e_{\sigma^{-i} b^{-1}} \\
& =\left(\sum_{i=0}^{3} r_{i} e_{\sigma^{-i}}\right)^{\eta(\gamma(b))}=\theta(s)^{\eta(\gamma(b))},
\end{aligned}
$$

while under $\theta^{\prime}=\operatorname{id}_{S}$ and $\gamma^{\prime}=\operatorname{id}_{G}$, we have the relation $\theta^{\prime}\left(s^{\eta(b)}\right)=\theta^{\prime}(s)^{\eta\left(\gamma^{\prime}(b)\right)}$ preserved, and $\gamma^{\prime}$ is trivial on $B$.

\section{Baer Products and Descent for Relative Embedding Problems}

From now on we restrict our attention to abelian relative embedding problems. Consider an embedding problem associated to $S_{B} / R, \eta_{S_{B}}: H \stackrel{\simeq}{\longrightarrow} \operatorname{Aut}\left(S_{B} / R\right)$, and $f: G \rightarrow H$; a Galois subalgebra $R_{1} / R$ with quotient action $\eta_{R_{1}}: H / \operatorname{Fix}\left(R_{1}\right) \stackrel{\simeq}{\longrightarrow}$ Aut $\left(R_{1} / R\right)$; and a solution $\left(T / R, \eta_{T}\right)$ to an embedding problem with group $G$, associated to $\left(R_{1} / R, \eta_{R_{1}}\right)$ and the quotient surjection $f^{\prime}: G \rightarrow H / \operatorname{Fix}\left(R_{1}\right)$, such that under $\eta_{T}$ the subgroup in $G$ corresponding to $R_{1}$ is abelian and contains ker $f$. We call the subalgebra $R_{1} / R$ the common subalgebra of $T / R$ and $S_{B} / R$.

Definition 2.1. The A-embedding problem associated to an abelian relative embedding problem is the embedding problem associated to $R_{1} / R, \eta_{R_{1}}: H / \operatorname{Fix}\left(R_{1}\right)$ $\stackrel{\cong}{A} \operatorname{Aut}\left(R_{1} / R\right)$, and $f^{\prime}: G \rightarrow H / \operatorname{Fix}\left(R_{1}\right)$. Denote ker $f^{\prime}$ by $A$. The $A$-embedding problem associated to an abelian relative embedding problem has a solution $\left(T / R, \eta_{T}\right)$. The split $A$-embedding problem is the embedding problem associated to $\left(R_{1} / R, \eta_{R_{1}}, A \rtimes G / A \stackrel{\text { can }}{\longrightarrow} G / A \cong H / \operatorname{Fix}\left(R_{1}\right)\right)$.

Definition 2.2. The $B$-embedding problem associated to an abelian relative embedding problem is the (original) embedding problem associated to $S_{B} / R, \eta_{S_{B}}: H$ $\stackrel{\simeq}{\rightarrow} \operatorname{Aut}\left(S_{B} / R\right)$, and $f: G \rightarrow H$. Denote ker $f$ by $B$. Solutions of the $B$-embedding problem associated to an abelian relative embedding problem are solutions of the relative embedding problem, and vice versa. All solutions of the $B$-embedding problem are also then solutions of the $A$-embedding problem.

Definition 2.3. The $A / B$-embedding problem associated to an abelian relative embedding problem is the embedding problem associated to $R_{1} / R, \eta_{R_{1}}: H / \operatorname{Fix}\left(R_{1}\right)$ $\stackrel{\simeq}{\rightarrow} \operatorname{Aut}\left(R_{1} / R\right)$, and $f^{\prime \prime}: H \rightarrow H / \operatorname{Fix}\left(R_{1}\right)$. Keeping the same notation, the kernel of $f^{\prime \prime}$ is then $A / B$. The $A / B$-embedding problem associated to an abelian relative embedding problem has a solution $S_{B} / R$. The split $A / B$-embedding problem is the embedding problem associated to $\left(R_{1} / R, \eta_{R_{1}}, A / B \rtimes G / A \stackrel{\text { can }}{\longrightarrow} G / A \cong H / \operatorname{Fix}\left(R_{1}\right)\right)$.

Let $C(A)$ denote the centralizer of $A$ in $G$. In the following, note that since $A$ is normal in $G, C(A)$ is also normal in $G$ and therefore $C(A) / A$ is normal in $G / A$.

Definition 2.4. The reduced embedding problems associated to an abelian relative embedding problem are as follows, and all but the last are necessarily split. Let $R_{0}$ be the fixed subalgebra of $C(A) / A \subset G / A$ in $R_{1}$ and denote by $\eta_{R_{0}}: H / \operatorname{Fix}\left(R_{0}\right) \stackrel{\simeq}{\longrightarrow}$ $\operatorname{Aut}\left(R_{0} / R\right)$ the quotient action. The reduced $A$-embedding problem is the embedding problem associated to $\left(R_{0} / R, \eta_{R_{0}}, A \rtimes G / C(A) \stackrel{\text { can }}{\longrightarrow} G / C(A) \cong H / \operatorname{Fix}\left(R_{0}\right)\right)$. The reduced $A / B$-embedding problem is the embedding problem associated to 
$\left(R_{0} / R, \eta_{R_{0}}, A / B \rtimes G / C(A) \stackrel{\mathrm{can}}{\longrightarrow} G / C(A) \cong H / \operatorname{Fix}\left(R_{0}\right)\right)$. Given a solution $\left(U_{0}, \eta_{U_{0}}\right)$ of the reduced $A / B$-embedding problem, the reduced $B$-embedding problem is the embedding problem associated to $\left(U_{0} / R, \eta_{U_{0}}, A \rtimes G / C(A) \stackrel{\text { can }}{\longrightarrow} A / B \rtimes G / C(A)\right)$.

Proposition 2.5 ([ILF, §3.15]). Fix the solution $\left(T / R, \eta_{T}\right)$ of the A-embedding problem. Then there exists a bijective correspondence between equivalence classes of solutions $\left(S / R, \eta_{S}\right)$ of the A-embedding problem and equivalence classes of solutions $\left(\tilde{U} / R, \eta_{\tilde{U}}\right)$ of the split $A$-embedding problem.

Proof ([ILF]). Let $\left(T / R, \eta_{T}\right)$ and $\left(S / R, \eta_{S}\right)$ be solutions of the $A$-embedding problem, hence $G$-Galois extensions. Let $i: \bar{G} \rightarrow G$ be the isomorphism carrying the Baer group inverse $\bar{G}$ of $G$ to $G$. Then $\left(T / R, \eta_{T} i\right)$ is a $\bar{G}$-Galois extension. We form the Baer product of Galois extensions $\left(T / R, \eta_{T} i\right)$ and $\left(S / R, \eta_{S}\right)$ as follows. Let $M$ be the $k$-algebra $T \otimes_{R_{1}} S$, with group the amalgamated product of $\bar{G}$ and $G$ over $H$, and with action $\eta_{M}=\eta_{T} i \otimes \eta_{S}$. The Baer product is then the subalgebra $\tilde{U}$ of $M$ fixed by $\eta_{M}\left(a, a^{-1}\right), a \in A$, which is the same as the subalgebra of elements of $M$ fixed by $\eta_{T}(a) \otimes \eta_{S}(a), a \in A$; the group is then the Baer product of $\bar{G}$ and $G$, namely the semidirect product $A \rtimes G / A$; and the action $\eta_{\tilde{U}}: A \rtimes G / A \stackrel{\simeq}{\longrightarrow} \operatorname{Aut}(\tilde{U} / R)$ is derived from $\eta_{M}$. Since $\left(T / R, \eta_{T}\right)$ and $\left(S / R, \eta_{S}\right)$ both extend $\left(R_{1} / R, \eta_{R_{1}}\right)$, the extension $\tilde{U} / R$ solves the embedding problem stated in the proposition.

Now let $\left(\tilde{U} / R, \eta_{\tilde{U}}\right)$ be an $A \rtimes G / A$-Galois extension which extends $\left(R_{1} / R, \eta_{R_{1}}\right)$, and let $\left(T / R, \eta_{T}\right)$ be a solution of the $A$-embedding problem. We form the Baer product of Galois extensions $\left(\tilde{U} / R, \eta_{\tilde{U}}\right)$ and $\left(T / R, \eta_{T}\right)$ as follows. Let $M$ be the $k$-algebra $\tilde{U} \otimes_{R_{1}} T$, with group the amalgamated product of $A \rtimes G / A$ and $G$ over $H$, and with action $\eta_{M}=\eta_{\tilde{U}} \otimes \eta_{T}$. The Baer product is then the subalgebra $S$ of $M$ fixed by $\eta_{M}\left(a, a^{-1}\right), a \in A$, which is the set of elements of $M$ fixed by $\eta_{\tilde{U}}(a) \otimes \eta_{T}\left(a^{-1}\right), a \in A$; the group is then the Baer product of $A \rtimes G / A$ and $G$, namely $G$; and the action $\eta_{S}: G \stackrel{\simeq}{\longrightarrow} \operatorname{Aut}(S / R)$ is derived from $\eta_{M}$. Since $\left(\tilde{U} / R, \eta_{\tilde{U}}\right)$ and $\left(T / R, \eta_{T}\right)$ both extend $\left(R_{1} / R, \eta_{R_{1}}\right)$, the extension $S / R$ solves the embedding problem stated in the proposition.

We now show that the correspondence is bijective. Having fixed $T / R$, we constructed a $\tilde{U} / R$ corresponding to any given $S / R$; we must show that the composition of this correspondence with the one which takes $\tilde{U} / R$ to a solution $S^{\prime} / R$ is the identity map. Consider the Galois extension

$$
N=T \otimes_{R_{1}} S \otimes_{R_{1}} T
$$

with group $G_{N}$, the amalgamated product of $\bar{G}, G$, and $G$ over $H$, with action $\eta_{N}=\eta_{T} i \otimes \eta_{S} \otimes \eta_{T}$. The composition of the two mappings gives a certain Galois subextension $S^{\prime} / R$ inside the Galois extension $N$, namely that associated under $\eta_{N}$ to the subgroup $J$ of $G_{N}$ generated by elements $\left(a, 1, a^{-1}\right)$ and $\left(a, a^{-1}, 1\right), a \in A$. Now set $\eta_{N}^{\prime}=\eta_{T} \otimes \eta_{S} \otimes \eta_{T}$ and view $\eta_{N}^{\prime}$ as an action of $A \times A \times A$ on the Galois extension $N / R_{1}$. Then $S^{\prime} / R_{1}$ is the subextension associated under $\eta_{N}^{\prime}$ to the subgroup $J^{\prime}$ of $A \times A \times A$ generated by elements $(a, 1, a)$ and $\left(a, a^{-1}, 1\right), a \in A$.

We must now show that the fixed subextension $S^{\prime} / R$, together with the quotient action given by $\eta_{N}$, is equivalent to $\left(S, \eta_{S}\right)$. First we determine the subextension of $T \otimes_{R_{1}} T$ with action $\eta_{T} \otimes \eta_{T}$ fixed by elements $(a, a)$. Following [ILF, lemma in Theorem 3.15.2], the algebra $T \otimes_{R_{1}} T$ with action $\eta_{T} \otimes \eta_{T}$ can be written as a sum $\bigoplus_{a \in A} T e_{1}$, where $e_{1}^{\eta_{T}(g) \otimes \eta_{T}(g)}=e_{1}$ for each $g \in G ; e_{a}=e_{1}^{\eta_{T}(a) \otimes \eta_{T}(1)}$; and $G$ acts on $T$ via the first factor in $\eta_{T} \otimes \eta_{T}$. Since $A$ is abelian, we have, moreover, that 
$e_{a}^{\eta_{T}(b) \otimes \eta_{T}(b)}=e_{a}$ for each $b \in A$. With these relations one derives the fact that the subalgebra of $T \otimes_{R_{1}} T$ fixed by elements $\eta_{T}(a) \otimes \eta_{T}(a), a \in A$, is $\bigoplus_{a \in A} R_{1} e_{a}$; further, the action of $A^{\prime}$, the factor group of $A \times A$ by elements of the form $(a, a)$, is via the same action $\eta_{T} \otimes \eta_{T}$, so that $a^{\prime} \in A^{\prime}$ acts as $\left(a^{\prime}, 1\right) \in A \times A$, or, equivalently, by permuting the summands,

$$
\sum_{a \in A} r_{a} e_{a} \mapsto \sum_{a \in A} r_{a} e_{a a^{\prime}}
$$

Then the subalgebra $S^{\prime}$ of $N$ is the subalgebra of $\left(\bigoplus_{a \in A} R_{1} e_{a}\right) \otimes_{R_{1}} S$ with action $\left(\left(\eta_{T} \otimes \eta_{T}\right) \otimes \eta_{S}\right)$ fixed by elements $\left((a, 1), a^{-1}\right), a \in A$. This tensor product can be written $\bigoplus_{a \in A} S e_{a}$, and the fixed elements are those of the form $\sum_{a \in A} z^{\eta_{S}\left(a^{-1}\right)} e_{a}$, $z \in S$. The group $A$, as a factor group of $A^{\prime} \times A$ by elements $\left(a, a^{-1}\right)$, then acts on $S^{\prime}$ as $((a, 1), 1)$ in $\left(\left(\eta_{T} \otimes \eta_{T}\right) \otimes \eta_{S}\right)$, so that $a^{\prime} \in A$ sends $\sum_{a \in A} z^{\eta_{S}\left(a^{-1}\right)} e_{a}$ to

$$
\sum_{a \in A} z^{\eta_{S}\left(a^{-1}\right)} e_{a a^{\prime}}=\sum_{a \in A}\left(z^{\eta_{S}\left(a^{\prime}\right)}\right)^{\eta_{S}\left(a^{-1}\right)} e_{a} .
$$

This algebra is then clearly isomorphic, with the action of $A$, to $S$ with action $\eta_{S}$, via $\sum_{a \in A} z^{a^{-1}} e_{a} \mapsto z$.

Corollary 2.6. Fix the solution $\left(T / R, \eta_{T}\right)$ of the A-embedding problem, and take the fixed subalgebra of $T / R$ corresponding to $B$, with quotient action: $\left(T_{B}, \eta_{T_{B}}\right)$. Then there exists a bijective correspondence between equivalence classes of solutions $\left(S_{B} / R, \eta_{S_{B}}\right)$ of the $A / B$-embedding problem and equivalence classes of solutions $\left(U_{1} / R, \eta_{U_{1}}\right)$ of the split $A / B$-embedding problem.

Proof. Let $(T, G, A)$ of the proposition refer to $\left(T_{B}, G / B, A / B\right)$; then the statement is the same, mutatis mutandis, as the proposition.

Proposition 2.7. Fix a relative embedding problem. Then there exists a bijective correspondence between equivalence classes of solutions $\left(S / R, \eta_{S}\right)$ of the $B$ embedding problem and equivalence classes of solutions $\left(\tilde{U} / R, \eta_{\tilde{U}}\right)$ of the embedding problem associated to the surjection

$$
A \rtimes G / A \rightarrow A / B \rtimes G / A \cong \operatorname{Gal}\left(U_{1} / R\right) .
$$

Here the extension $\left(U_{1} / R, \eta_{U_{1}}\right)$ corresponds to $\left(S_{B} / R, \eta_{S_{B}}\right)$ under Corollary 2.6.

Proof. Solutions of the $B$-embedding problem are also solutions of the $A$-embedding problem, and hence we may invoke Proposition 2.5 to assert that solutions $\left(S / R, \eta_{S}\right)$ of the $B$-embedding problem correspond to solutions $\left(\tilde{U}, \eta_{\tilde{U}}\right)$ of the split $A$-embedding problem. By Corollary 2.6, the extension $S_{B} / R$ of the relative embedding problem corresponds to a solution $\left(U_{1} / R, \eta_{U_{1}}\right)$ of the $A / B$-embedding problem. Since the correspondences are achieved in the same way and $\left(S / R, \eta_{S}\right)$ extends $\left(S_{B} / R, \eta_{S_{B}}\right),\left(\tilde{U} / R, \eta_{\tilde{U}}\right)$ extends $\left(U_{1} / R, \eta_{U_{1}}\right)$ and hence $\left(\tilde{U} / R, \eta_{\tilde{U}}\right)$ is a solution of the embedding problem associated to $A \rtimes G / A \rightarrow A / B \rtimes G / A \cong \operatorname{Gal}\left(U_{1} / R\right)$.

Conversely, again by the identical process of the correspondences, we have that given a solution $\left(\tilde{U} / R, \eta_{\tilde{U}}\right)$ of the embedding problem associated to $\left(U_{1} / R, \eta_{U_{1}}\right)$ and surjection $A \rtimes G / A \rightarrow A / B \rtimes G / A$, which is then a solution of the split $A$-embedding problem, we have that by Proposition $2.5\left(\tilde{U} / R, \eta_{\tilde{U}}\right)$ corresponds to a solution $\left(S / R, \eta_{S}\right)$ of the $A$-embedding problem, and, using Corollary 2.6, we find that this solution must extend the solution $\left(S_{B} / R, \eta_{S_{B}}\right)$ corresponding to $\left(U_{1} / R, \eta_{U_{1}}\right)$. 
Proposition 2.8. Fix an abelian relative embedding problem. Then there exists a bijective correspondence between solutions $\left(\tilde{U} / R, \eta_{\tilde{U}}\right)$ of the split A-embedding problem and solutions $\left(U / R, \eta_{U}\right)$ of the reduced $A$-embedding problem.

Proof. Let $\left(\tilde{U} / R, \eta_{\tilde{U}}\right)$ be a solution of the split $A$-embedding problem. The subgroup $C(A)$ is normal in $G$, hence $C(A) / A$ is normal in $G / A$. Therefore the subgroup $1 \rtimes C(A) / A$ of the semidirect product $A \rtimes G / A$ is normal. Taking the fixed ring of this subgroup under $\eta_{\tilde{U}}$ in $\tilde{U}$, we have a solution $\left(U / R, \eta_{U}\right)$ of the reduced $A$-embedding problem, where $\eta_{U}$ is the quotient action from $\eta_{\tilde{U}}$.

Conversely, note that subalgebra $R_{1} / R$ of the abelian relative embedding problem is a $G / A$-extension with action $\eta_{R_{1}}$ derived from the solution $\left(T, \eta_{T}\right)$. Now let $\left(U / R, \eta_{U}\right)$ be a solution of the reduced $A$-embedding problem, and consider the $k$-algebra $N=U \otimes_{R_{0}} R_{1}$, where $R_{0}$ is the fixed subalgebra of $C(A) / A$ in $R_{1}$, with action $\eta_{N}=\eta_{U} \otimes \eta_{R_{1}}$. This $k$-algebra $N$ is a Galois extension over $R$ with group isomorphic to the product of $A \rtimes G / C(A)$ (under action $\eta_{U}$ ) and $G / A$ (under action $\eta_{R_{1}}$ ) with amalgamated quotient group $G / C(A)$, under an action, say $\eta_{R_{0}}$, which is common to $\eta_{U}$ and $\eta_{R_{1}}$ by virtue of the definition of the reduced $A$-embedding problem. This amalgamation is a group $\tilde{H}$ which is a semidirect product of $A$ with $G / A$, hence $\left(N, \eta_{N}\right)$ is a solution of the split $A$-embedding problem. Set $\left(\tilde{U}, \eta_{\tilde{U}}\right)$ equal to $\left(N, \eta_{N}\right)$.

We now show that the correspondence is bijective. Given a solution $\left(U / R, \eta_{U}\right)$ of the reduced $A$-embedding problem, we see that the corresponding solution $\left(\tilde{U} / R, \eta_{\tilde{U}}\right)$ has subalgebra $U / R$ corresponding to $1 \rtimes C(A) / A$ under the action $\eta_{\tilde{U}}$. Hence the correspondence $\tilde{U} \mapsto U$ results in the same $\left(U / R, \eta_{U}\right)$.

Corollary 2.9. Fix an abelian relative embedding problem. Then there exists a bijective correspondence between solutions $\left(U_{1} / R, \eta_{U_{1}}\right)$ of the split $A / B$-embedding problem and solutions $\left(U_{0} / R, \eta_{U_{0}}\right)$ of the reduced $A / B$-embedding problem.

Proof. Let $\left(T_{B}, \eta_{T_{B}}\right)$ be the fixed subalgebra of the solution $\left(T, \eta_{T}\right)$ of the $A$ embedding problem, under $B$ and $\eta_{T}$; the quotient action is then $\eta_{T_{B}}$. Let $(T, G, A)$ of the proposition refer to $\left(T_{B}, G / B, A / B\right)$; then the statement is the same, mutatis mutandis, as the proposition.

Proposition 2.10. Fix an abelian relative embedding problem. Then there exists a bijective correspondence between equivalence classes of solutions $\left(S / R, \eta_{S}\right)$ of the $B$-embedding problem and equivalence classes of solutions $\left(U / R, \eta_{U}\right)$ of the reduced $B$-embedding problem associated to the extension $\left(U_{0} / R, \eta_{U_{0}}\right)$ corresponding to $\left(S_{B} / R, \eta_{S_{B}}\right)$ under Corollary 2.9 .

Proof. By Proposition 2.7, solutions $\left(S / R, \eta_{S}\right)$ correspond to solutions $\left(\tilde{U} / R, \eta_{\tilde{U}}\right)$, and by Proposition 2.8 this solution corresponds to a solution $\left(U / R, \eta_{U}\right)$. By Proposition $2.7\left(\tilde{U} / R, \eta_{\tilde{U}}\right)$ extends $\left(U_{1} / R, \eta_{U_{1}}\right)$, which corresponds in the same sense as Proposition 2.8 under Corollary 2.9 to $\left(U_{0} / R, \eta_{U_{0}}\right)$. Hence $\left(U / R, \eta_{U}\right)$ extends $\left(U_{0} / R, \eta_{U_{0}}\right)$ and solves the reduced $A / B$-embedding problem. The converse follows just as in the proof of Proposition 2.7.

Proposition 2.11. Assume that the $k$-algebras in the abelian relative embedding problem are fields and $S_{B}$ and $T$ are linearly disjoint over $R_{1}$. Then the correspondences in Propositions 2.5, 2.7, 2.8, and 2.10 and Corollaries 2.6 and 2.9 carry field solutions to field solutions as follows: 
(a) Proposition 2.5: solutions $S$ linearly disjoint from $T$ over $R_{1} \longleftrightarrow$ solutions $\tilde{U}$ linearly disjoint from $T$ over $R_{1}$;

(b) Corollary 2.6: solutions $S_{B}$ linearly disjoint from $T$ over $R_{1} \longleftrightarrow$ solutions $U_{1}$ linearly disjoint from $T$ over $R_{1}$;

(c) Proposition 2.7: solutions $S$ linearly disjoint from $T$ over $R_{1} \longleftrightarrow$ solutions $\tilde{U}$ linearly disjoint from $T$ over $R_{1}$;

(d) Proposition 2.8: solutions $\tilde{U} \longleftrightarrow$ solutions $U$ linearly disjoint from $R_{1}$ over $R_{0}$;

(e) Corollary 2.9: solutions $U_{1} \longleftrightarrow$ solutions $U_{0}$ linearly disjoint from $R_{1}$ over $R_{0}$

(f) Proposition 2.10: solutions $S$ linearly disjoint from $T$ over $R_{1} \longleftrightarrow$ solutions $U$ linearly disjoint from $R_{1}$ over $R_{0}$.

Proof. For (a), (b), and (c), observe that the Baer product of two Galois field extensions linearly disjoint over $R_{1}$ is again linearly disjoint over $R_{1}$ from either extension. In (d) and (e) one direction is given by taking a subfield and does not depend on the Baer product, while the other one is taken via the Baer product with $R_{1} / R$. The last is the combination of (a) through (e).

\section{Main Theorem and Applications to Dihedral Field Extensions}

In this section we consider the abelian relative embedding problem in the setting of fields. We change notation for the field case and then specialize Proposition 2.10 to our Main Theorem.

Definition 3.0. The abelian relative embedding problem for fields is defined as follows. Let $G$ be a finite group with a normal abelian subgroup $A$, and assume that $B$ is a subgroup of $A$ which is also normal in $G$. Let $K$ be a field and $L / K$ a $G$-Galois extension where we identify $G$ with the Galois group of $L / K$. Suppose that $E_{B} / K$ is a $G / B$-Galois extension such that $L \cap E_{B}=K_{1}$, where $K_{1} / K$ is a Galois extension corresponding to $A \subset G$. Let $\dot{G}$ be an abstract group isomorphic to $G$, such that $\dot{G} \rightarrow G / B$ is the surjection corresponding to $G \rightarrow G / B$. The abelian relative embedding problem is to determine all $\dot{G}$-Galois extensions $E / K$ which extend $E_{B} / K$.

In the following main theorem we connect the solutions of the relative abelian embedding problem for fields and the solutions of a reduced embedding problem for fields.

Theorem 3.1 (Main Theorem: Reduction). Consider an abelian relative embedding problem for fields as defined above. Let $K_{0}$ be the fixed field in $L$ of the centralizer $C(A)$ of $A$ in $G$, which is also the fixed field of $C(A) / A$ in $K_{1}$. Then

(a) $E_{B} / K$ corresponds uniquely to a solution $F_{0} / K$ of the embedding problem associated to the split exact sequence

$$
1 \longrightarrow A / B \longrightarrow A / B \rtimes G / C(A) \longrightarrow G / C(A) \cong \operatorname{Gal}\left(K_{0} / K\right) \longrightarrow 1 .
$$

Here $G / C(A)$ acts on $A / B$ via the action of $G$ on $A$; and

(b) the solutions $E / K$ of the relative embedding problem which are linearly disjoint from $L$ over $K_{1}$ are in bijective correspondence with the solutions $F / K$ of the embedding problem associated to the exact sequence

$$
1 \longrightarrow B \rtimes 1 \longrightarrow A \rtimes G / C(A) \longrightarrow A / B \rtimes G / C(A) \cong \operatorname{Gal}\left(F_{0} / K\right) \longrightarrow 1
$$

which are linearly disjoint from $K_{1}$ over $K_{0}$. 
Proof. With notation changed as in Definition 3.0 and attention paid to linear disjointness using Proposition 2.11, the theorem is a specialization of Proposition 2.10 to fields.

Now we specialize our Main Theorem to a Dihedral Reduction Theorem for fields for the case in which $G$ is a dihedral group. Let $C_{s}$ denote the cyclic group of order $s$ and $D_{s} \cong C_{s} \rtimes C_{2}$ the dihedral group of order $2 s$. Let $1<k|m| n$ be integers and set $G=D_{n}$.

We set up the abelian relative embedding problem and reinterpret Theorem 3.1 as follows. Let $L / K$ be a Galois extension with $\operatorname{Gal}(L / K) \cong D_{n}$. Let $E_{B} / K$ be a Galois extension with $\operatorname{Gal}\left(E_{B} / K\right) \cong D_{m}$, such that $E_{B} \cap L=K_{1}$ is Galois with $\operatorname{Gal}\left(K_{1} / K\right) \cong D_{k}$. Note that in this situation $A \cong C_{n / k}, B \cong C_{n / m}, C(A) \cong C_{n}$, and the fixed field of $C(A)$ in $L$ is $K_{0}$.

Since $L / K_{0}$ is a cyclic Galois extension with a group $C_{n}$, there is a unique $C_{m^{-}}$Galois subextension $L_{B} / K_{0}$ and it contains $K_{1}$. Furthermore, $E_{B}$ and $L_{B}$ are $C_{m / k}$-Galois extensions of $K_{1}$, thus corresponding to two distinct elements, $\alpha$ and $\beta$, respectively, of the cohomology group $H^{1}\left(K_{1}, C_{m / k}\right)$. There exists a unique $C_{m / k^{-}}$-Galois extension of $K_{1}$ corresponding to the element $\alpha \beta^{-1} \in H^{1}\left(K_{1}, C_{m / k}\right)$. We denote this extension of $K_{1}$ by $F_{1}$.

The thrust of Theorem 3.1(a) is that $F_{1} / K_{1}$ descends to a $C_{m / k}$-Galois extension $F_{0}$ of $K_{0}$ (so that, in particular, $F_{1}=F_{0} \otimes_{K_{0}} K_{1}$ ); further, $F_{0} / K$ is a Galois extension with group $C_{m / k} \rtimes C_{2}$, with a quotient action derived from $G$ : $F_{0} / K$ is Galois with group $D_{m / k}$, cyclic over $K_{0}$. The content of Theorem 3.1(b) is then that $E_{B} / K$ embeds in a $D_{n}$-extension of $K$ cyclic over $K_{0}$ if and only if the extension $F_{0} / K$ embeds in a $D_{m}$-extension of $K$ cyclic over $K_{0}$. Note that if $m>2$, then the cyclic over $K_{0}$ condition is automatically satisfied for a $D_{n}$-extension of $K$ extending $E_{B} / K$, and if $m / k>2$, then the cyclic over $K_{0}$ condition is automatically satisfied for a $D_{m}$-extension of $K$ extending $F_{0} / K$.

Theorem 3.2 (Dihedral Reduction). Let $k, m, n$ be integers as above and let all Galois extensions denote those of field extensions. Let $L / K$ be a $D_{n}$-Galois extension and $E_{B} / K$ a $D_{m}$-Galois extension such that $L \cap E_{B}=K_{1}$ is a Galois extension of $K$ with group $D_{k}$.

Then there exists a $D_{m / k}$-Galois extension $F_{0} / K$, cyclic over $K_{0}$, such that $E_{B} / K$ embeds in a $D_{n}$-extension $E / K$ cyclic over $K_{0}$ if and only if the $D_{m / k^{-}}$ Galois extension $F_{0} / K$ embeds in a $D_{n / k}$-Galois extension of $K$ cyclic over $K_{0}$.

Proof. Let $L / K$ be a $D_{n}$-Galois extension with group presented as

$$
\left\langle\sigma, \tau: \sigma^{n}=1, \tau^{2}=1, \tau \sigma \tau=\sigma^{-1}\right\rangle,
$$

and let $E_{B} / K$ be $D_{m}$-Galois extension with group presented as

$$
\left\langle\hat{\sigma}, \hat{\tau}: \hat{\sigma}^{m}=1, \hat{\tau}^{2}=1, \hat{\tau} \hat{\sigma} \hat{\tau}=\hat{\sigma}^{-1}\right\rangle,
$$

such that $K_{1}=E_{B} \cap L$ is a $D_{k}$-extension of $K$ corresponding to the fixed fields of $\left\langle\sigma^{k}\right\rangle \subset D_{n}$ and $\left\langle\hat{\sigma}^{k}\right\rangle \subset D_{m}$, where $\sigma$ and $\hat{\sigma}$ (respectively $\tau$ and $\hat{\tau}$ ) act identically on $K_{1}$. Let $L_{B}$ be the fixed field of $\left\langle\sigma^{m}\right\rangle$ in $L$.

Let $F_{1}$ be the fixed field of the elements $\left(\sigma^{k i}, \hat{\sigma}^{k i}\right)$ in the compositum $E_{B} L_{B}$ for $0 \leq i<m / k$. Then $F_{1} / K$ is the unique $C_{m / k}$-Galois extension of $K_{1}$ corresponding to the Baer product (of Galois extensions) of $E_{B} / K$ and the inverse of $L_{B} / K$. The subgroup $\langle\sigma\rangle \subset \operatorname{Gal}\left(K_{1} / K\right)$ lifts to a normal subgroup of $\operatorname{Gal}\left(F_{1} / K\right)$, generated by $\sigma \in \operatorname{Gal}\left(K_{1} / K\right)$ extended to $\operatorname{Gal}\left(F_{1} / K_{1}\right)$ as $(\sigma, \hat{\sigma})$. Let $F_{0}$ be the fixed field in $F_{1}$ of 
this subgroup; it is the unique extension described in Theorem 3.1(a). By Theorem 3.1(b), then, letting $A=\left\langle\sigma^{k}\right\rangle$ and $B=\left\langle\sigma^{m}\right\rangle$, we have that $E_{B} / K$ embeds in a $D_{n}$-extension $E / K$ cyclic over $K_{0}$, i.e., in which $\hat{\tau}$ and $\hat{\sigma} \hat{\tau}$ are lifted to elements of order 2 , if and only if the $D_{m / k}$-extension $F_{0}$ of $K$ embeds in a $D_{n / k}$-extension of $K$ cyclic over $K_{0}$, with group

$$
\left\langle\tilde{\sigma}, \tilde{\tau}: \tilde{\sigma}^{n / k}=1, \tilde{\tau}^{2}=1, \tilde{\tau} \tilde{\sigma} \tilde{\tau}=\tilde{\sigma}^{-1}\right\rangle,
$$

where $\tau$ lifts to $\tilde{\tau}$.

Now we specialize even further to the case of dihedral groups of 2-power order and field $K$ of characteristic not 2. Suppose $L / K$ is a Galois extension with $\operatorname{Gal}(L / K) \cong$ $D_{2^{d}}$ and let $K_{0} / K$ be the unique quadratic subextension corresponding to the fixed field in $L$ of the cyclic subgroup $C_{2^{d}}$. Denote a generator of $C_{2^{d}}$ in Galois $\operatorname{group} \operatorname{Gal}(L / K)$ by $\sigma$. Let $L_{i}$ be the fixed field in $L$ of $\left\langle\sigma^{\left(2^{i}\right)}\right\rangle$ for $i=1, \ldots, d$. Thus the set $\left\{L_{i} / K\right\}$ may be viewed as a tower of dihedral Galois extensions, with $\operatorname{Gal}\left(L_{i} / K\right) \cong D_{2^{i}}$.

It is well-known that all embeddings of $L_{i} / K$ into $D_{2^{i+1}}$-Galois extensions of $K$ cyclic over $K_{0}$ "differ" from $L_{i+1}$ by a square root of an element in $K^{\times}$(see for example [Bl, Lemma 4.1]); more precisely, if $L_{i+1}=L_{i}\left(\sqrt{\gamma_{i}}\right)$ for some $\gamma_{i} \in L_{i}$, then all embeddings of $L_{i} / K$ into $D_{2^{i+1}}$-Galois extensions cyclic over $K_{0}$ are of the form $L_{i}\left(\sqrt{r \gamma_{i}}\right)$ for $r \in K^{\times}$, and any $L_{i}\left(\sqrt{r \gamma_{i}}\right)$ is such an embedding. In the following theorem we determine the condition on $r$ permitting such alternate $D_{2^{i+1}}$-Galois extensions to embed into $D_{2^{i+j}}$-Galois extensions cyclic over $K_{0}$; the condition is the solvability of a reduced embedding problem, which can be thought of as a quotient of the embedding problems extending $L_{i+1}$ and $L_{i}\left(\sqrt{r \gamma_{i}}\right)$, in the sense of our section 4 . As stated above, if $i>1$, then the cyclic over $K_{0}$ condition is automatically satisfied.

Theorem 3.3 (Dihedral 2-Group Reduction). Assume that the characteristic of the field $K$ is different from 2. Let $L / K$ be a $D_{2^{d}}$-Galois extension of fields, with $L_{i}$ defined as above. Let $K_{0}=K(\sqrt{a b})$ and $L_{1}=K_{0}(\sqrt{a})$ for $a, b \in K^{\times}$. Choose $\gamma_{i} \in L_{i}$ such that $L_{i+1}=L_{i}\left(\sqrt{\gamma_{i}}\right)$ for $i=1, \ldots, d-1$. Let $r \in K^{\times} \backslash K^{\times^{2}}$ such that $r, a, b$, and $a b$ are independent in $K^{\times} / K^{\times^{2}}$.

Then for $j=1, \ldots, d-i$, the $D_{2^{i}}$-Galois field extension $L_{i}$ embeds in a $D_{2^{i+j}-}$ Galois field extension which extends $L_{i}\left(\sqrt{r \gamma_{i}}\right)$ and is cyclic over $K_{0}$ if and only if the $D_{2}$-Galois field extension $K_{0}(\sqrt{r}) / K$ embeds in a $D_{2^{j}}$-Galois field extension cyclic over $K_{0}$.

Proof. We apply Theorem 3.2 with $n=2^{i+j}, m=2^{i+1}, k=2^{i}$; we must only determine $F_{0} / K$. We have that $E_{B}=L_{i}\left(\sqrt{r \gamma_{i}}\right)$ and $L_{B}=L_{i+1}=L_{i}\left(\sqrt{\gamma_{i}}\right)$. The compositum $E_{B} L_{B}$ is then $L_{i}\left(\sqrt{\gamma_{i}}, \sqrt{r}\right)$, and the Baer product of $E_{B}$ and the inverse of $L_{B}$ over $L_{i}$ is then $L_{i}(\sqrt{r})$, which is then $F_{1}$. Now $F_{0} / K$ is a $D_{2}$-Galois extension extending $K_{0} / K=K(\sqrt{a b}) / K$ inside $F_{1} / K$. Because $F_{1}=L_{i} \otimes_{K} K(\sqrt{r})$, we have that $\operatorname{Gal}\left(F_{1} / K\right) \cong D_{2^{i}} \times C_{2}$, and the $D_{2}$-Galois subextensions are the $C_{2} \times C_{2}$-Galois subextensions of the $C_{2} \times C_{2} \times C_{2}$-Galois subextension $K(\sqrt{a}, \sqrt{b}, \sqrt{r})$. By the discussion above Theorem 3.2 we know that $F_{1}=F_{0} \otimes_{K_{0}} L_{i}$, and since $L_{i}$ contains the subextension $K(\sqrt{a}, \sqrt{b})$, we must have that $F_{0}$ is $K_{0}(\sqrt{r s})=K_{0}(\sqrt{r s a b})$ for $s=1$ or $a$. But since $F_{1} / L_{i}$ descends to $F_{0} / K_{0}$, we have that $s=1$, i.e., $F_{0}=K_{0}(\sqrt{r})=K(\sqrt{r}, \sqrt{a b})$. 
Examples 3.4: Explicit 2-Power Dihedral Extensions. For embedding problems consisting of small 2-power dihedral groups, the obstructions to their solution (see section 4) and some formulas for the explicit construction of their solution fields are well-known. We use these obstructions and our reduction theorems to describe some explicit 2-power dihedral extensions over fields $K$ of characteristic not 2 .

Examples 3.4.1 $\left(D_{2^{v}} \rightarrow D_{4}\right)$. It is known (from GSS, Proposition 3.10], for instance) that any $D_{4}$-Galois extension $W$ of such a field $K$ is of the form $W=$ $K(\sqrt{x+y \sqrt{a}}, \sqrt{b})$, where $a$ and $b$ are independent in $K^{\times} / K^{\times^{2}}$ and there exists $z \in K^{\times}$such that $x^{2}-a y^{2}=b z^{2}$. The extension $W / K$ is then cyclic over $K(\sqrt{a b})$.

Let $E_{B}=K(\sqrt{r(x+y \sqrt{a})}, \sqrt{b})$ for $r \in K^{\times} \backslash K^{\times^{2}}$ an element such that $r, a$, $b$, and $a b$ are independent in $K^{\times} / K^{\times 2}$. Now suppose that $W$ embeds in a $D_{2^{v}}$ extension $L$ of $K$. Then by Theorem 3.3, $E_{B}$ embeds in a $D_{2^{v}}$-extension $E$ of $K$ if and only if $N=K(\sqrt{r}, \sqrt{a b})$ embeds in a $D_{2^{v-1}}$-extension of $K$ cyclic over $K(\sqrt{a b})$.

Example 3.4.2 $\left(D_{16} \rightarrow D_{8}\right)$. First we describe all admissible $D_{8}$-extensions in the sense of [Sw1], applying the transformation described after [GSS, Theorem 4.5.2] correctly; note that this corrects an error in [GSS, Theorem 4.5.3] and describes all $D_{8}$-extensions over fields $K=\mathbb{Q}$ and $K=\mathbb{Q}(t)$.

Let $L_{B}=K(\chi, \sqrt{b})$, where

$$
\chi=\sqrt{s\left(\tilde{z}+\frac{y}{2} \sqrt{a}\right)\left(2 x+2\left(c+\frac{a e y^{2}}{4}-\frac{e x y}{4} \sqrt{a}\right) \sqrt{x+y \sqrt{a}}\right)} .
$$

Here $a$ and $b$ are independent $\bmod K^{\times 2} ; s \in K^{\times} ; x, y$, and $z$ satisfy $x^{2}-a y^{2}=b z^{2}$ as above; $\tilde{z}, w \in K$ are such that $\tilde{z}^{2}-2 w^{2}=a y^{2} / 4 ; c, e \in K$ are such that $c^{2}+\left(a b y^{2} \tilde{z}^{2} / 16\right) e^{2}=x / 2 ; z \neq 0 ; w \neq 0 ; z \neq w$; and $z \neq a y^{2} /(2 x)$. Then $L_{B}$ is a $D_{8}$-extension of $K$ extending $W$, and all $D_{8}$-extensions of $\mathbb{Q}$ or $\mathbb{Q}(t)$ may be so described for suitable $W$.

Second, we describe an $E_{B}$ resulting from a solvable embedding problem in Example 3.4.1. Let $r$ be such that the embedding problem for $K(\sqrt{r}, \sqrt{a b})$ and $D_{4} \rightarrow$ $D_{2}$ is solvable with a solution field cyclic over $K(\sqrt{a b})$, as in Example 3.4.1. Then, by [GSS, Proposition 3.10], the quaternion algebra $(r,-a b)_{K}$ must be split. Applying [GSS Theorem 4.5.3] to $L_{B} / K$, the quaternion algebra $(2 x,-a b)_{K}$ is split. Viewing the quaternion algebras as Hilbert symbols, we have $(r,-a b)(2 x,-a b) \sim$ $(2 x r,-a b) \sim(r x / 2,-a b)$. Hence there exist $c^{\prime}, e^{\prime} \in K$ satisfying

$$
{c^{\prime}}^{2}+\left(a b(r y)^{2}(r z)^{2} / 16\right) e^{\prime 2}=r x / 2 .
$$

The $D_{8}$-extensions $E_{B} / K$ which solve the problem of Example 3.4.1 are then described with $x^{\prime}=r x, y^{\prime}=r y, z^{\prime}=r z, \tilde{z}^{\prime}=r \tilde{z}, w^{\prime}=r w$, and $E_{B}=K\left(\sqrt{\chi^{\prime}}, \sqrt{b}\right)$, where

$$
\chi^{\prime}=\sqrt{s^{\prime}\left(\tilde{z}^{\prime}+\frac{y^{\prime}}{2} \sqrt{a}\right)\left(2 x^{\prime}+2\left(c^{\prime}+\frac{a e^{\prime} y^{\prime 2}}{4}-\frac{e^{\prime} x^{\prime} y^{\prime}}{4} \sqrt{a}\right) \sqrt{x^{\prime}+y^{\prime} \sqrt{a}}\right)},
$$

$s^{\prime} \in K^{\times}$. Let $\hat{\sigma}$ be a generator of the $C_{8}$-subgroup of $\operatorname{Gal}\left(E_{B} / K\right)$, and let $\hat{\tau} \in$ $\operatorname{Gal}\left(E_{B} / K\right)$ leave $\chi^{\prime}$ invariant. 
Now suppose that $L_{B}$ embeds in a $D_{16}$-extension of $K$. One shows, using the relations for admissible dihedral extensions in [Sw1], that the element

$$
\nu=\chi \chi^{\prime}+\chi^{\sigma} \chi^{\prime \hat{\sigma}}+\chi^{\sigma^{2}} \chi^{\prime \hat{\sigma}^{2}}+\chi^{\sigma^{3}} \chi^{\prime \hat{\sigma}^{3}}
$$

lies in $F_{0}$ and has square

$$
32 s s^{\prime} r^{2} x^{2} \tilde{z}^{2}+q^{\prime} \sqrt{r}
$$

for $q^{\prime} \in K$. Therefore the element $\omega=\nu /(4 r x \tilde{z}) \in N$ has square $2 s s^{\prime}+q \sqrt{r}$ for $q \in K$. Then we have that

$$
F_{0}=K\left(\sqrt{2 s s^{\prime}+q \sqrt{r}}, \sqrt{a b}\right)
$$

for $q \in K^{\times}$and we have found the $F_{0}$ such that $E_{B}$ embeds in a $D_{16}$-extension of $K$ if and only if $F_{0}$ embeds in a $D_{8}$-extension of $K$.

\section{Applications to Obstructions to Central $C_{p}$-Embedding Problems}

When the embedding problem under consideration is central with kernel isomorphic to $C_{p}$ over the field $k$ containing $p$-th roots of unity, one associates to the problem an element of $p$-torsion component of the Brauer group, known as the obstruction. This element often determines the precise solvability conditions of the associated embedding problem; see Remark 4.2 below. In this section we explore some of the implications of our Main Theorem in the theory of obstructions.

From our Main Theorem we deduce that the condition determining the solvability of an abelian relative embedding problem is identical to the condition determining the solvability of a reduced embedding problem. In the following Theorem 4.3 we make this statement precise in the context of central Galois embedding problems with kernel $C_{p}$ over a $k$-algebra containing the $p$-th roots of unity, proving that the reduced embedding problem can be viewed as a quotient of two embedding problems, and, moreover, that the obstruction of the reduced embedding problem is the quotient of the obstructions to the two associated embedding problems. Thus, even when it is not known that one of the embedding problems is solvable, we have that the reduced embedding problem expresses the condition by which the two embedding problems differ. Then, in Theorem 4.6, we show how a phenomenon related to this connection gives information about the structure of the obstructions, particularly when the obstructions can be expressed as tensor products of $p$-cyclic algebras. In what follows, we denote the Brauer group of a ring $R$ by $\operatorname{Br}(R)$.

Definition 4.1. Let $p$ be a prime and $k$ a field of characteristic not $p$ containing the full group $\mu_{p}$ of $p$-th roots of unity. Let $\left(S_{B} / R, \eta_{S_{B}}\right)$ be an $H$-Galois extension of $k$-algebras. Let $f: G \rightarrow H$ be a surjection of groups where $B=\operatorname{ker} f$ is central and isomorphic to $C_{p}$. Then the obstruction $O_{S_{B} / R}=O_{S_{B} / R, \eta_{S_{B}}, f \text { of the embedding }}$ problem $\left(S_{B} / R, \eta_{S_{B}}, f\right)$ is the class $\left[\left(S_{B} / R, \eta_{S_{B}}, \overline{c_{f}}\right)\right] \in \operatorname{Br}(R)$ of the crossed product $\left(S_{B} / R, \eta_{S_{B}}, \overline{c_{f}}\right)$, where $\overline{c_{f}} \in H^{2}\left(H, \mu_{p} \cong B\right)$ is a 2-cocycle describing the $C_{p^{-}}$ extension $G$ of $H$.

Remark 4.2. If $R$ is a field $K$, then in some situations the obstruction is a "proper" obstruction, i.e., the embedding problem has a (proper) solution if and only if the obstruction vanishes in $\operatorname{Br}(K)$, which means that the class of the crossed product is trivial. One situation is when the embedding problem is Frattini. Another is when the field $K$ is Hilbertian, by a result of Ikeda [Ik]. The obstruction is also 
useful in constructing the solutions of the embedding problem: given an explicit isomorphism from a matrix ring over $K$ to a representative of the Brauer class, one has a method explicitly to construct all of the solution fields Sw2.

Theorem 4.3. Let $p$ be a prime and $k$ a field of characteristic not $p$ containing the full group $\mu_{p}$ of $p$-th roots of unity. Let $G$ be a group with a normal abelian subgroup $A$ containing a subgroup $B \cong C_{p}$ lying in the center of $G$. Let $H=G / B$, and let $\left(S_{B} / R, \eta_{S_{B}}\right)$ and $\left(T_{B} / R, \eta_{T_{B}}\right)$ be two $H$-Galois extensions (of algebras) which are common over $\left(R_{1} / R, \eta_{R_{1}}\right)$, the fixed subalgebra with quotient action of $\eta_{S_{B}}(A / B)$ in $S_{B}$ and of $\eta_{T_{B}}(A / B)$ in $T_{B}$.

Then, as elements of $\operatorname{Br}(R)$,

$$
O_{S_{B}, \eta_{S_{B} / R}, G \rightarrow G / B} O_{T_{B} / R, \eta_{T_{B}}, G \rightarrow G / B}^{-1}=O_{U_{0} / R, \eta_{U_{0}}, A \rtimes G / C(A) \stackrel{\text { can }}{\longrightarrow} A / B \rtimes G / C(A)},
$$

where $\left(U_{0} / R, \eta_{U_{0}}\right)$ is the fixed subalgebra with quotient action of $1 \rtimes C(A) / A$ in the $A / B \rtimes G / A$-Galois extension $\left(U_{1} / R, \eta_{U_{1}}\right)$, which in turn is given by the Baer product of the Galois extension $\left(S_{B}, \eta_{S_{B}}\right)$ and the inverse of the Galois extension $\left(T_{B}, \eta_{T_{B}}\right)$.

Proof. Let $N=S_{B} \otimes_{R_{1}} T_{B}$, with group $\Gamma$, be the amalgamated product of $H$ and $\bar{H}$ over $G / A$, and action $\eta_{N}=\eta_{S_{B}} \otimes \eta_{T_{B}} i$, where $i$ is the isomorphism from the Baer inverse $\bar{H}$ of $H$ over $G / A$ to $H$. Then the Baer product $\left(U_{1} / R, \eta_{U_{1}}\right)$ is the fixed subalgebra of $N$ corresponding to the subgroup $(A / B)^{\sim}$ consisting of elements $\left(a, \bar{a}^{-1}\right), a \in A / B$, and is an $A / B \rtimes G / A$-Galois extension. Note that in this proof we emphasize elements of $\bar{H}$ by denoting them with a bar.

Let $f: G \rightarrow H=G / B$ denote the surjection of the embedding problems associated to $S_{B} / R$ and $T_{B} / R$, and let $r: A \rtimes G / C(A) \rightarrow A / B \rtimes G / C(A)$ denote the surjection of the embedding problem associated to $U_{0} / R$. We consider $\inf _{N / S_{B}} c_{f}, \inf _{N / T_{B}} c_{f}^{-1}$, and $\inf _{N / U_{0}} c_{r}$ in the cohomology group $H^{2}\left(\eta_{N}(\Gamma), \mu_{p}\right)$, where $\eta_{N}(\Gamma)$ acts trivially on $\mu_{p}$. Note that in order to make precise the inflation maps, we must specify the maps between actions which are to take place; these are the maps, respectively, $\eta_{N}(\Gamma) \rightarrow \eta_{S_{B}}(H), \eta_{N}(\Gamma) \rightarrow\left(\eta_{T_{B}} i\right)(\bar{H})$, and $\eta_{N}(\Gamma) \rightarrow \eta_{U_{1}}(A / B \rtimes G / A) \rightarrow \eta_{U_{0}}(A / B \rtimes G / C(A))$, of Galois theory.

The inflation map $\inf _{N / S_{B}}$ corresponds to amalgamation with $\left(T_{B} / R, \eta_{T_{B}} i\right)$. More precisely, the map takes the class of an extension of the $H$-Galois extension $\left(S_{B} / R, \eta_{S_{B}}\right)$ by an extension with group $B$ and sends it to the class of the amalgamation of this extension with the $H$-Galois extension $\left(T_{B} / R, \eta_{T_{B}} i\right)$ over the common subextension $\left(R_{1} / R, \eta_{R_{1}}\right)$. Hence $\inf _{N / S_{B}} c_{f}$ describes a group $\Gamma_{1}$ which is the amalgamation of $G$ with the Baer inverse of $G / B$, over the common factor group $G / A$, where the kernel of $\Gamma_{1} \rightarrow \Gamma$ commutes with elements in $A / B \subset\left(\eta_{T_{B}} i\right)(\bar{H})$. In 2-cocycle notation, this map is expressed as follows:

$$
\left(\inf _{N / S_{B}} c_{f}\right)\left(\left(h_{1}, \overline{h_{2}}\right),\left(h_{3}, \overline{h_{4}}\right)\right)=c_{f}\left(h_{1}, h_{3}\right), \quad\left(h_{1}, \overline{h_{2}}\right),\left(h_{3}, \overline{h_{4}}\right) \in \Gamma .
$$

Similarly, the inflation map $\inf _{N / T_{B}}$ corresponds to amalgamation with $\left(S_{B} / R, \eta_{S_{B}}\right)$. Thus, $\inf _{N / T_{B}} c_{f}^{-1}$ describes a group $\Gamma_{2}$ which is the amalgamation of $G / B$ with $\bar{G}$, over the common factor group $G / A$, where the kernel of $\Gamma_{2} \rightarrow \Gamma$ commutes with elements in $A / B \subset \eta_{S_{B}}(H)$. In 2-cocycle notation, this map is expressed as follows:

$$
\left(\inf _{N / T_{B}} c_{f}^{-1}\right)\left(\left(h_{1}, \overline{h_{2}}\right),\left(h_{3}, \overline{h_{4}}\right)\right)=c_{f}^{-1}\left(i\left(\overline{h_{2}}\right), i\left(\overline{h_{4}}\right)\right), \quad\left(h_{1}, \overline{h_{2}}\right),\left(h_{3}, \overline{h_{4}}\right) \in \Gamma .
$$


By [ILF, Theorem 3.15.1], the multiplication of classes in $H^{2}\left(\eta_{N}(\Gamma), \mu_{p}\right)$ corresponds on one hand to function multiplication, and on the other to the Baer product of the group extensions corresponding to the classes, where the amalgamation occurs over $\Gamma$. Hence the quotient $\left(\inf _{N / S_{B}} c_{f}\right)\left(\inf _{N / T_{B}} c_{f}^{-1}\right)$ describes the Baer product $\tilde{\Gamma}$ of $\Gamma_{1}$ and $\Gamma_{2}$ over $\Gamma$. Since $\Gamma$ is the amalgamated product of $H$ and $\bar{H}$ over $G / A$, and since $\Gamma_{1}$ is the amalgamation of $G$ and $\bar{H}$ over $G / A$, and $\Gamma_{2}$ is the amalgamation of $H$ and $\bar{G}$ over $G / A$, we have that $\tilde{\Gamma}$ is the quotient of the amalgamation of $G$ and $\bar{G}$ over $G / A$ by the set of elements $\left(b, \bar{b}^{-1}\right), b \in B$.

By the definition of the Baer product, we have a canonical surjection $p: \tilde{\Gamma} \rightarrow \Gamma$. Consider $p^{-1}\left((A / B)^{\sim}\right) \subset \tilde{\Gamma}$. Let $\left(a_{1}, \bar{a}_{1}^{-1}\right),\left(a_{2}, \bar{a}_{2}^{-1}\right) \in(A / B)^{\sim}$. By function multiplication of 2-cocycles in $Z^{2}\left(\eta_{N}(\Gamma), \mu_{p}\right)$ we have that

$$
\begin{aligned}
& \left(\inf _{N / S_{B}} c_{f} \cdot \inf _{N / T_{B}} c_{f}^{-1}\right)\left(\left(a_{1},{\overline{a_{1}}}^{-1}\right),\left(a_{2},{\overline{a_{2}}}^{-1}\right)\right) \\
& \quad=\left(c_{f}\left(a_{1}, a_{2}\right) \cdot c_{f}^{-1}\left(i\left({\overline{a_{1}}}^{-1}\right), i\left({\overline{a_{2}}}^{-1}\right)\right)\right)=c_{f}\left(a_{1}, a_{2}\right) c_{f}^{-1}\left(a_{1}, a_{2}\right)=1 .
\end{aligned}
$$

Therefore, when restricted to $(A / B)^{\sim}$, the group extension $\tilde{\Gamma}$ of $\Gamma$ corresponds to $(A / B)^{\sim} \times B$. Hence the subgroup $(A / B)^{\sim} \times 1$ of $\tilde{\Gamma}$ is isomorphic to $(A / B)^{\sim}$ and is a preimage of $(A / B)^{\sim}$ under $p$. By abuse of notation we denote this subgroup $(A / B)^{\sim} \subset \tilde{\Gamma}$.

We now consider the quotient of $\tilde{\Gamma}$ by $(A / B)^{\sim}$. Because $\tilde{\Gamma}$ is the quotient of the amalgamated product of $G$ and $\bar{G}$ over $G / A$ by the elements $\left(b, \bar{b}^{-1}\right), b \in B$, and the elements in $(A / B)^{\sim}$ are of the form $\left(a, \bar{a}^{-1}\right), a \in A / B$, we have that $\tilde{\Gamma} /(A / B)^{\sim}$ is the quotient of the amalgamated product of $G$ and $\bar{G}$ over $G / A$ by the elements $\left(a, \bar{a}^{-1}\right), a \in A$, or, in other words, the Baer product of $G$ and $\bar{G}$ over $G / A$, which is the semidirect product $A \rtimes G / A$. Furthermore, we may view $(A / B)^{\sim}$ as the group of the Galois subextension $\left(N / U_{1}, \eta_{N}\right)$, so that $\Gamma /(A / B)^{\sim}$ is the group of the Galois extension $\left(U_{1} / R, \eta_{U_{1}}\right)$ and $\tilde{\Gamma}$ is the group extension of $\Gamma$ given by the surjection $A \rtimes G / A \stackrel{\text { can }}{\longrightarrow} A / B \rtimes G / A$. Indeed, the group $\tilde{\Gamma}$ appears by amalgamation of $\tilde{\Gamma} /(A / B)^{\sim}$ with the Galois extension $\left(N / U_{1}, \eta_{N}\right)$.

On the other hand, $r$ describes the group extension problem $A \rtimes G / C(A) \stackrel{\text { can }}{\longrightarrow}$ $A / B \rtimes G / C(A)$. Let $R_{0}$ be the fixed subalgebra of $C(A) / A$ in the $G / A$-Galois extension $\left(R_{1}, \eta_{R_{1}}\right)$. Then $U_{1}=U_{0} \otimes_{R_{0}} R_{1}$ and $\eta_{U_{1}}=\eta_{U_{0}} \otimes \eta_{R_{1}}$. By the argument in Proposition 2.7 the inflation $c_{r}^{\prime}=\inf _{U_{1} / U_{0}} c_{r}$ sends this group extension to the group extension $A \rtimes G / A \stackrel{\text { can }}{\longrightarrow} A / B \rtimes G / A$. By the previous paragraph $\inf _{N / U_{1}} c_{r}^{\prime}$ describes $\tilde{\Gamma}$, so we are done.

Examples 4.4: Cyclic 2-Power Obstructions over Fields. In each of the following cases we set $B=C_{2}$ and consider central $C_{2}$-extensions over a field $R=$ $K=k$ of characteristic not 2 . We use the notation for fields established at the beginning of section 3 .

Example 4.4.1 $\left(C_{8} \rightarrow C_{4}\right.$ over $\left.C_{2}\right)$. Let $G=C_{8}=\langle\sigma\rangle, A=\left\langle\sigma^{2}\right\rangle, B=\left\langle\sigma^{4}\right\rangle$. Then $H=G / B \cong C_{4}$. It is well-known that $H$-Galois extensions $E_{B} / K$ and $L_{B} / K$ common over the $G / A$-extension $K_{1} / K=K(\sqrt{d}) / K$ are all of the form

$$
K(\sqrt{r d+r y \sqrt{d}})
$$


$r \in K^{\times}$, where there exist $x, y \in K^{\times}$such that $x^{2}+y^{2}=d \in K^{\times} \backslash K^{\times^{2}}$ (see, for example, GSS, Proposition 3.4]). Let

$$
E_{B} / K=K\left(\sqrt{r_{1} d+r_{1} y \sqrt{d}}\right) / K
$$

and

$$
L_{B} / K=K\left(\sqrt{r_{2} d+r_{2} y \sqrt{d}}\right) / K,
$$

where $r_{1}, r_{2}$, and $d$ are necessarily independent in $K^{\times} / K^{\times 2}$. Then $F_{0} / K=$ $K\left(\sqrt{r_{1} r_{2}}\right) / K$. By [SC] we have that $O_{E_{B} / K}=(2, d)\left(-1, r_{1}\right)$, where the latter is the class of a tensor product of quaternion algebras over $K ; O_{L_{B} / K}=(2, d)\left(-1, r_{2}\right)$; and $O_{F_{0} / K}=\left(-1, r_{1} r_{2}\right)$. Then Theorem 4.3 gives us the following relation in $\operatorname{Br}(K)$ :

$$
\left((2, d)\left(-1, r_{1}\right)\right)\left((2, d)\left(-1, r_{2}\right)\right)^{-1}=\left(-1, r_{1} r_{2}\right) .
$$

Example 4.4.2 $\left(C_{16} \rightarrow C_{8}\right.$ over $C_{2}$ for $K=\mathbb{Q}$ or $\left.\mathbb{Q}(t)\right)$. Let $G=C_{16}=\langle\sigma\rangle, A=$ $\left\langle\sigma^{2}\right\rangle, B=\left\langle\sigma^{8}\right\rangle$. Then $H=G / B \cong C_{8}$. By [Sw1, all $H$-Galois extensions $E_{B} / K$ and $L_{B} / K$ common over the $G / A$-extension $K_{1} / K=K(\sqrt{d}) / K$ which are admissible in the sense of [Sw1] are all of the form

$$
K\left(\sqrt{s(z+\sqrt{d})\left(2 r d+v \psi+u \psi^{\sigma}\right)}\right),
$$

for $r, s \in K^{\times}$, where $\psi=\left(r d+r y d^{1 / 2}\right)^{1 / 2}$; there exist $x, y \in K^{\times}$such that $x^{2}+y^{2}=$ $d \in K^{\times} \backslash K^{\times 2} ; z, w \in K$ such that $z^{2}-2 w^{2}=d$; and $t_{1}, t_{2} \in K$ such that $t_{1}^{2}+t_{2}^{2}=r$; and $u=t_{1} x-t_{2} y-t_{1} y-t_{2} x, v=t_{1} x-t_{2} y+t_{1} y+t_{2} x$. Note that in our case the $x, y, z$, and $w$ may be fixed for both $E_{B}$ and $L_{B}$. By [Sc], all $H$-Galois extensions over $\mathbb{Q}$ and $\mathbb{Q}(t)$ are admissible.

Let

$$
E_{B} / K=K\left(\sqrt{s_{1}(z+\sqrt{d})\left(2 r_{1} d+v_{1} \psi_{1}+u_{1} \psi_{1}^{\sigma}\right)}\right) / K
$$

and

$$
L_{B} / K=K\left(\sqrt{s_{2}(z+\sqrt{d})\left(2 r_{2} d+v_{2} \psi_{2}+u_{2} \psi_{2}^{\sigma}\right)}\right) / K,
$$

where $r_{1}, r_{2}$, and $d$ are necessarily independent in $K^{\times} / K^{\times 2} ; r_{1}=t_{1,1}^{2}+t_{1,2}^{2}$; $r_{2}=t_{2,1}^{2}+t_{2,2}^{2}$; and $u_{1}, u_{2}, v_{1}$, and $v_{2}$ are defined analogously. Then one shows that

$$
F_{0} / K=K\left(\sqrt{s_{1} s_{2} r_{1} r_{2}+s_{1} s_{2}\left(t_{1,1} t_{2,1} \pm t_{1,2} t_{2,2}\right) \sqrt{r_{1} r_{2}}}\right) / K
$$

Using [Sw1] for the computation of obstructions, we have that

$$
O_{E_{B} / K}=\left(s_{1}(z-w),-1\right)\left(r_{1} z(z-y),-2\right)(z(z-w), d),
$$

where the latter is the class of a tensor product of three quaternion algebras over $K$

$$
O_{L_{B} / K}=\left(s_{2}(z-w),-1\right)\left(r_{2} z(z-y),-2\right)(z(z-w), d) ;
$$


and $O_{F_{0} / K}=\left(-1, s_{1} s_{2}\right)\left(2, r_{1} r_{2}\right)$. Then Theorem 4.3 gives us the following relation in $\operatorname{Br}(K)$ :

$$
\begin{aligned}
& \left(\left(s_{1}(z-w),-1\right)\left(r_{1} z(z-y),-2\right)(z(z-w), d)\right) \\
& \quad \cdot\left(\left(s_{2}(z-w),-1\right)\left(r_{2} z(z-y),-2\right)(z(z-w), d)\right)^{-1} \\
& \quad=\left(-1, s_{1} s_{2}\right)\left(-2, r_{1} r_{2}\right)=\left(-1, s_{1} s_{2}\right)\left(2, r_{1} r_{2}\right) .
\end{aligned}
$$

The last equality holds since

$$
\left(-1, r_{1}\right)=\left(-1, r_{2}\right)=\left(-1, r_{1} r_{2}\right)=1
$$

We now introduce the concept of a relatively general pair of embedding problems and prove a structural statement about obstructions of relatively general pairs, followed by an example of this phenomenon.

Definition 4.5. Let $p$ be a prime and $k$ a field of characteristic not $p$ containing the full group $\mu_{p}$ of $p$-th roots of unity. Let $G$ be a finite group with normal abelian subgroup $A$, containing a normal subgroup $B \cong C_{p}$.

Suppose that $\left(S_{B} / R, \eta_{S_{B}}\right)$ is a $G / B$-Galois extension and $\left(U_{0} / R, \eta_{U_{0}}\right)$ is an $A / B \rtimes G / C(A)$-Galois extension, common over the $G / C(A)$-Galois extension $\left(R_{0} / R, \eta_{R_{0}}\right)$, the fixed subalgebra with quotient action of $\eta_{S_{B}}(C(A) / B)$ in $S_{B}$ and $\eta_{U_{0}}(A / B \rtimes 1)$ in $U_{0}$. Let the $G / A$-Galois extension $\left(R_{1} / R, \eta_{R_{1}}\right)$ be the fixed subalgebra with quotient action of $\eta_{S_{B}}(A / B)$ in $S_{B}$. Let $\left(T_{B} / R, \eta_{T_{B}}\right)$ denote the Baer product of $\left(S_{B} / R, \eta_{S_{B}}\right)$ and $\left(\left(U_{0} \otimes_{R_{0}} R_{1}\right) / R, \eta_{U_{0}} \otimes \eta_{R_{1}}\right)$ over $\left(R_{1} / R, \eta_{R_{1}}\right)$.

Then $\left(S_{B} / R, \eta_{S_{B}}\right)$ and $\left(U_{0} / R, \eta_{U_{0}}\right)$ form a relatively general pair if there exists an injection $\alpha: R \rightarrow R$ and an $R_{1}$-isomorphism $\beta: S_{B} \otimes_{\alpha} R \rightarrow T_{B}$ such that $\beta\left(s^{\eta_{S_{B}}(g)}\right)=\beta(s)^{\eta_{T_{B}}(g)}$ for $s \in S_{B}, g \in G / B$.

Theorem 4.6. Let $\left(S_{B} / R, \eta_{S_{B}}\right)$ and $\left(U_{0} / R, \eta_{U_{0}}\right)$ form a relatively general pair. Then

$\alpha\left(O_{S_{B} / R, \eta_{S_{B}}, G \rightarrow G / B}\right) O_{S_{B} / R, \eta_{S_{B}}, G \rightarrow G / B}^{-1}=O_{U_{0} / R, \eta_{U_{0}}, A \rtimes G / C(A) \stackrel{\text { can }}{\longrightarrow} A / B \rtimes G / C(A)}$, where $\alpha$ acts on the crossed product via $\left(\cdot \otimes_{\alpha} R\right)$ on $S_{B}$ and $\operatorname{id}_{G / B}$ on $G / B$.

Proof. By Theorem 4.3 we need only show that the two crossed products

$$
O_{T_{B} / R, \eta_{T_{B}}, G \rightarrow G / B}, \quad \alpha\left(O_{S_{B} / R, \eta_{S_{B}}, G \rightarrow G / B}\right)
$$

are $R$-isomorphic, and this isomorphism is given by extending the $\beta$ of the definition of a relatively general pair by the identity on the elements of $G / B$ in the crossed product.

Example 4.7: $D_{8} \rightarrow D_{4}$. Let $k$ be a field of characteristic not 2. As in Example 3.4 .1 , it is well-known that $D_{4}$-extensions over a field $K$ of characteristic not 2 are of the form $K(\sqrt{x+y \sqrt{a}}, \sqrt{b}) / K$, for a choice of $a, b \in K$ such that $a$ and $b$ are independent in $K^{\times} / K^{\times 2}$, and there exist $x, y, z \in K$ such that $x^{2}-a y^{2}-b z^{2}=0$. Let

$$
R=k[a, b, x, y, z, r](1 / a b r x y z) /\left\langle x^{2}-a y^{2}-b z^{2}\right\rangle .
$$

Note that $R$ is étale over a localized polynomial ring $k[a, b, x, y, z, r](1 / a b r x y z)$, and thus $R$ is normal, i.e. integrally closed in its field of fractions. Next, let

$$
S_{B} / R=R(\sqrt{x+y \sqrt{a}}, \sqrt{b}) / R
$$


Note that $S_{B}$ is étale over $R$ and thus is normal. Since the field extension corresponding to fields of fractions of $S_{B} / R$ is a $D_{4}$-Galois extension, we have that $S_{B} / R$ is a $D_{4}$-Galois extension. Let

$$
G=D_{8}=\left\langle\sigma, \tau: \sigma^{8}=\tau^{2}=1, \tau \sigma \tau^{-1}=\sigma^{-1}\right\rangle,
$$

and let $A=\langle\sigma\rangle, B=\left\langle\sigma^{2}\right\rangle$. Then the corresponding reduced embedding problem is that of $D_{4} \rightarrow D_{2}$, and we choose the $D_{2}$-Galois extension $U_{0} / R=R(\sqrt{a b}, \sqrt{r}) / R$. One shows that

$$
T_{B} / R=R(\sqrt{r x+r y \sqrt{a}}, \sqrt{b}) / R
$$

Let $\alpha: R \rightarrow R$ be given by $\alpha(a)=a, \alpha(b)=b, \alpha(r)=r, \alpha(x)=r x, \alpha(y)=r y$, $\alpha(z)=r z$; then $\alpha$ and $\beta=$ id show that $S_{B} / R$ and $U_{0} / R$ are a relatively general pair. We have that

$$
O_{S_{B} / R, \eta_{S_{B}}, D_{8} \rightarrow D_{4}}=(a, 2)(2 x,-a b)
$$

where the latter denotes the tensor product of a pair of quaternion algebras, and

$$
O_{U_{0} / R, \eta_{U_{0}}, D_{4} \rightarrow D_{2}}=(r,-a b) \text {. }
$$

Applying Theorem 4.6, we recover

$$
((a, 2)(2 x r,-a b))((a, 2)(2 x,-a b))^{-1}=(r,-a b) .
$$

\section{Applications to $p$-Metacyclic Embedding Problems: DETERMINING TOWERS}

Constructing large dihedral extensions of a field remains a problem of interest, and if one uses the theory of embedding problems, one naturally seeks to "climb up" a tower of dihedral extensions, constructing them iteratively. The case of dihedral towers is a particularly attractive context for the theory of relative embedding problems since the existence of infinite towers of dihedral extensions implies that results such as Theorem 3.3 have a fairly general application. In order to find similarly general contexts, we are led to consider other infinite towers of groups for which our Main Theorem is applicable. In this section we classify, for all $p$, all towers which are analogous in a certain sense: given a fixed metacyclic nonabelian $p$-group $G$ with normal cyclic subgroup $A$ and cyclic quotient $G / A$, we determine all towers of metacyclic $p$-groups over $G$ which extend the cyclic group $A$. We do not treat the case of abelian groups, since the many towers over a given abelian group which contain a nonabelian member are distinguished by their smallest nonabelian group. In determining the towers, we also specify the corresponding groups which occur in the reduced embedding problems.

We first recall a description of metacyclic $p$-groups which is particularly useful for our purposes, due to Liedahl [Li]. Let $G$ be a metacyclic $p$-group of order $p^{N}$. Then $G$ can be specified by a quintuple $(c, n, m, t, s)$ with parameters limited to the following three types, where in every case $n+m=N$ :

Case $1(c=1 ; p$ odd):

$$
\begin{gathered}
G \cong\left\langle x, y \mid x^{p^{n}}=1, y^{p^{m}}=x^{p^{s}}, y x y^{-1}=x^{(p+1)^{p^{t}}}\right\rangle, \\
t \in\{\max (0, n-m-1), \cdots, n-1\}, \quad s \in\{n-t-1, \cdots, \min (n, m)\} ;
\end{gathered}
$$


Case $2(c=2 ; p=2)$ :

$$
\begin{gathered}
G \cong\left\langle x, y \mid x^{2^{n}}=1, y^{2^{m}}=x^{2^{s}}, y x y^{-1}=x^{5^{2^{t}}}\right\rangle, \\
t \in\{\max (0, n-m-2), \cdots, n-2\}, \quad s \in\{n-t-2, \cdots, \min (n, m)\} ;
\end{gathered}
$$

Case $3(c=3 ; p=2)$ :

$$
\begin{aligned}
& G \cong\left\langle x, y \mid x^{2^{n}}=1, y^{2^{m}}=x^{2^{s}}, y x y^{-1}=x^{-5^{2^{t}}}\right\rangle, \quad 2 \leq n \leq N-1, \\
& t \in\{\max (0, n-m-2), \cdots, n-2\}, \quad s \in\{n-1, \min (n, N-n+t+1)\} .
\end{aligned}
$$

These descriptions are not unique: as [Li, Remark 2.4.3] points out, even discounting the many presentations of the above types for groups of order $p$ and $p^{2}$, the metacyclic $p$-groups which are split by a cyclic subgroup may have multiple descriptions, and there are some groups which have presentations of both the second and third types. However, in the following proposition we insure that once we fix the normal subgroup $A=\langle x\rangle$, then there is a unique set of parameters $(c, n, m, t, s)$ above.

Proposition 5.1. Let $G$ be a nonabelian metacyclic p-group with normal cyclic subgroup $A$ and cyclic quotient group $G / A$. Then there exists one and exactly one presentation above for $G$ in which $\langle x\rangle=A$.

Proof. By [Li] we know that any nonabelian metacyclic $p$-group has a presentation listed above and that we may choose one such that $\langle x\rangle=A$. We must show then that if $\tilde{x}$ and $\tilde{y}$ are any two generators of $G$, with $\tilde{x} \in A$, then the resulting presentation of $G$ as $\langle\tilde{x}, \tilde{y}\rangle$ is identical to the first.

First, note that for any presentation above with generators $\tilde{x}$ and $\tilde{y}$, the pair $\hat{x}$ and $\tilde{y}$, where $\hat{x}$ is any element such that $\langle\tilde{x}\rangle=\langle\hat{x}\rangle$, generate $G$ with the same presentation as $\tilde{x}$ and $\tilde{y}$. Hence if $G$ has another presentation $G=\langle\tilde{x}, \tilde{y}\rangle$ with $\langle\tilde{x}\rangle=A$ in addition to the first presentation $G=\langle x, y\rangle$, then we may assume without loss of generality that $x=\tilde{x}$. We then must show that for no other choice of $\tilde{y} \in G$ such that $G=\langle x, \tilde{y}\rangle$ does the pair $x, \tilde{y}$ satisfy a different presentation as above.

Depending on the case of the first presentation, we have that $y x y^{-1}=x^{(p+1)^{p^{t}}}$

(in case 1), or $y x y^{-1}=x^{ \pm 5^{2^{t}}}$ (in cases 2 and 3). Consider a general element $\tilde{y}=x^{i} y^{j} \in G \backslash A$ with $0 \leq i<p^{n}$ and $0<j<p^{m}$. The conjugation relation between $x$ and $\tilde{y}$ becomes

$$
\tilde{y} x \tilde{y}^{-1}=\left(x^{i} y^{j}\right) x\left(x^{i} y^{j}\right)^{-1}=x^{(p+1)^{j p^{t}}}
$$

in case 1 and

$$
\tilde{y} x \tilde{y}^{-1}=x^{( \pm 1)^{j} 5^{j 2^{t}}}
$$

in cases 2 and 3 . Now if $(j, p) \neq 1$, then

$$
\tilde{y}^{p^{m-1}}=\left(x^{i} y^{j}\right)^{p^{m-1}} \in A,
$$

whence $\langle x, \tilde{y}\rangle \subsetneq G$. Moreover, since the presentations above restrict the exponent of $x$ in the conjugation relation to be a power of $p$, a power of 5 , or the negative of a power of 5 , we cannot have that $\tilde{y}=x^{i} y^{j}$ for some $j>1$ with $(j, p)=1$; hence no other choice of $\tilde{y}$ save $x^{i} y$ can serve as another possible generator with $x$. 
We then invoke [Li, §2.1], which insures that no two of the presentations above describe the same group under an isomorphism $x \mapsto x, y \mapsto x^{i} y$. Hence the only $\tilde{y}$ such that $G=\langle x, \tilde{y}\rangle$ under a presentation above is $\tilde{y}=y$. Once the normal cyclic subgroup $A=\langle x\rangle$ is fixed, then, the presentation of $G$ is unique.

In the following theorem and corollary we describe the set of towers of metacyclic $p$-groups which have a given nonabelian group $G$ as the smallest member and which only extend a fixed normal cyclic subgroup $A$. In the situation of Galois theory, then, if $G=\operatorname{Gal}(M / K)$ with $L=M^{A}$, then we show the possible Galois groups of Galois field extensions $\tilde{M} / K$ with degree $p^{N}$ such that $\operatorname{Gal}(\tilde{M} / L)$ is cyclic, extending $A=\operatorname{Gal}(M / L)$.

Theorem 5.2 (Towers). Let $G$ be a nonabelian metacyclic p-group with distinguished cyclic subgroup $A$, and let $(c, n, m, t, s)$ be its unique parameters as in Proposition 5.1. Consider the set of metacyclic p-groups $H$ such that there exists a surjection $f: H \rightarrow G$ which extends $\langle x\rangle$ in $G$, i.e., we have $(x \in H) \mapsto(x \in G)$ and $\operatorname{ker} f=\left\langle x^{p^{n}}\right\rangle$. Then the parameters as above for the set of such groups $H$, together with the centralizers $C_{H}(\langle x\rangle)$ and the quotient groups $H / C_{H}(\langle x\rangle)$, are as follows:

Case 1: If $s \neq n$, then $n^{\prime} \in\{n+1, \cdots, \min (t+m+1, s+t+1)\} ; m^{\prime}=m ; t^{\prime}=t$; $s^{\prime}=s ; C_{H}(\langle x\rangle)=\left\langle x, y^{p^{n^{\prime}-t-1}}\right\rangle ;$ and $H / C_{H}(\langle x\rangle) \cong C_{n^{\prime}-t-1}$.

If $s=n$, then $n^{\prime} \in\{n+1, \cdots, t+m+1\} ; m^{\prime}=m ; t^{\prime}=t ; s^{\prime} \in\left\{\max \left(n^{\prime}-t-\right.\right.$ $\left.1, n), \cdots, \min \left(n^{\prime}, m\right)\right\} ; C_{H}(\langle x\rangle)=\left\langle x, y^{p^{n^{\prime}-t-1}}\right\rangle ;$ and $H / C_{H}(\langle x\rangle) \cong C_{n^{\prime}-t-1}$.

Case 2: If $s \neq n$, then $n^{\prime} \in\{n+1, \cdots, \min (t+m+2, s+t+2)\} ; m^{\prime}=m ; t^{\prime}=t$; $s^{\prime}=s ; C_{H}(\langle x\rangle)=\left\langle x, y^{2^{n^{\prime}-t-2}}\right\rangle ;$ and $H / C_{H}(\langle x\rangle) \cong C_{n^{\prime}-t-2}$.

If $s=n$, then $n^{\prime} \in\{n+1, \cdots, t+m+2\} ; m^{\prime}=m ; t^{\prime}=t ; s^{\prime} \in\left\{\max \left(n^{\prime}-t-\right.\right.$ $\left.2, n), \cdots, \min \left(n^{\prime}, m\right)\right\} ; C_{H}(\langle x\rangle)=\left\langle x, y^{2^{n^{\prime}-t-2}}\right\rangle ;$ and $H / C_{H}(\langle x\rangle) \cong C_{n^{\prime}-t-2}$.

Case 3: If $s=n-1 \neq m+t+1$, then there are no such groups. If $s=n$ or $s=n-1=m+t+1$, and $t<n-2$, then $n^{\prime} \in\{n+1, \cdots, t+m+2\}$; $m^{\prime}=m ; t^{\prime}=t ; s^{\prime} \in\left\{n^{\prime}-1, \cdots, \min \left(n^{\prime}, m+t+1\right)\right\} ; C_{H}(\langle x\rangle)=\left\langle x, y^{2^{n^{\prime}-t-2}}\right\rangle ;$ and $H / C_{H}(\langle x\rangle) \cong C_{n^{\prime}-t-2}$.

If $s=n$ and $t=n-2$, then $n^{\prime}>n ; m^{\prime}=m ; t^{\prime} \in\left\{\max \left(n-2, n^{\prime}-m-2\right), \cdots\right.$, $\left.n^{\prime}-2\right\} ; s^{\prime} \in\left\{n^{\prime}-1, \cdots, \min \left(n^{\prime}, m+t^{\prime}+1\right)\right\} ; C_{H}(\langle x\rangle)=\left\langle x, y^{2}\right\rangle ;$ and $H / C_{H}(\langle x\rangle) \cong$ $\mathrm{C}_{2}$.

Corollary 5.3 (Infinite Towers). The infinite towers of nonabelian metacyclic pgroups extending a fixed normal cyclic subgroup $\langle x\rangle$ in the sense of Theorem 5.2 have as foundations the 2-groups of the following type:

$$
\left\langle x, y \mid x^{2^{n}}=1, y^{2^{m}}=1, y x y^{-1}=x^{-1}\right\rangle .
$$

If $A$ is a subgroup of $\langle x\rangle$, then $C(A)=\left\langle x, y^{2}\right\rangle$ and $G / C(A) \cong C_{2}$.

Proof of Theorem 5.2. Let $\left(c^{\prime}, n^{\prime}, m^{\prime}, t^{\prime}, s^{\prime}\right)$ be a description of a group $H$ satisfying the conditions of the theorem. Then under the surjection $f$, the elements $x \in H$ and $y \in H$ are sent to generators $f(x), f(y)$ of $G$, respectively, and these generators are associated to a presentation of $G$ related to that of $H$, with description $\left(c^{\prime \prime}, n^{\prime \prime}, m^{\prime \prime}, t^{\prime \prime}, s^{\prime \prime}\right)$. We study this description of $G$; then, based on the uniqueness of the presentation of $G$, we deduce restrictions on the presentation of $H$. The descriptions of $C_{H}(\langle x\rangle)$ and $H / C_{H}(\langle x\rangle)$ in the statement of the theorem are easily calculated. 
Assume that the presentation of $H$ falls into one of the first two cases. Then $G$ has a parametrization in the same case. First, $f(x)$ clearly has order $p^{n}$, so $n^{\prime \prime}=n$. Second, since $f(y)$ must have the same order as $y, m^{\prime \prime}=m^{\prime}$. Depending on the case, we have in $H$ the conjugation relation

$$
y x y^{-1}=x^{(p+1)^{p^{t^{\prime}}}}
$$

in case 1 , or

$$
y x y^{-1}=x^{5^{2^{t^{\prime}}}}
$$

in case 2 . Now the order of the automorphism $x \mapsto x^{p+1}$ is $p^{n-1}$ in case 1 , where $p^{n}$ is the order of $x$; similarly, the order of the automorphism $x \mapsto x^{5}$ is $2^{n-2}$ in case 2 . Hence if $t^{\prime} \geq n-1$ in case 1 or $t^{\prime} \geq n-2$ in case 2 , then we have that $G$ is abelian, which by hypothesis it is not, and otherwise we may take $t^{\prime \prime}=t^{\prime}$. Note that $t^{\prime \prime}$ lies within the appropriate bounds, because, in case 1 , if $n^{\prime}-m^{\prime}-1 \leq t^{\prime}$, then since $n \leq n^{\prime}$ and $m^{\prime \prime}=m^{\prime}$, we have $n^{\prime \prime}-m^{\prime \prime}-1 \leq t^{\prime \prime}$, and the analysis is the same for case 2 .

Again, depending on the case, we have that

$$
y^{p^{m^{\prime}}}=x^{p^{s^{\prime}}}
$$

in case 1 , or

$$
y^{2^{m^{\prime}}}=x^{2^{s^{\prime}}}
$$

in case 2 , so that if $s^{\prime} \geq n$ in either case, we may take $s^{\prime \prime}=n$, otherwise $s^{\prime \prime}=s^{\prime}$; hence $s^{\prime \prime}=\min \left(s^{\prime}, n\right)$. Note that $s^{\prime \prime}$ lies within the appropriate bounds, for $s^{\prime \prime} \leq$ $m^{\prime \prime}$, since if $m^{\prime \prime}<s^{\prime \prime}$, then $m^{\prime}<s^{\prime}$, which cannot occur in cases 1 and 2 , and, just as with $t^{\prime \prime}=t^{\prime}$ and $n^{\prime \prime}-m^{\prime \prime}-1, s^{\prime \prime}$ must be greater than $n^{\prime \prime}-t^{\prime \prime}-1$ in case 1 and $n^{\prime \prime}-t^{\prime \prime}-2$ in case 2 .

Now assume that the presentation of $H$ falls into the third case. Then $G$ has a parametrization in the same case. First, $f(x)$ clearly has order $p^{n}$, so $n^{\prime \prime}=n$. Second, since $f(y)$ must have the same order as $y, m^{\prime \prime}=m^{\prime}$. We have in $H$ the conjugation relation

$$
y x y^{-1}=x^{-5^{2^{t^{\prime}}}} .
$$

Now the order of the automorphism $x \mapsto x^{5}$ is $2^{n-2}$, where $2^{n}$ is the order of $x$; therefore, if $t^{\prime} \geq n-2$, then we may take $t^{\prime \prime}=n-2$. Otherwise, the same conjugation relation holds in $G$, so that $t^{\prime \prime}=t^{\prime}$. Combining both possibilities, $t^{\prime \prime}=\min \left(t^{\prime}, n-2\right)$.

We also have that in $H, y^{2^{m^{\prime}}}=x^{2^{s^{\prime}}}$, so that if $s^{\prime} \geq n$, we may take $s^{\prime \prime}=n$, and if not, $s^{\prime \prime}=s^{\prime}$. Combining both possibilities, we have that $s^{\prime \prime}=\min \left(s^{\prime}, n\right)$. As before, the value of $t^{\prime \prime}$ lies in the appropriate bounds for case 3 ; we need only check the bounds for $s^{\prime \prime}$. Note that the parametrization for case 3 states that either $s \in\{n-1, n\}$ or $s=n-1$, according to whether or not $m+t+1 \geq n$. Moreover, if $m+t+1=n-1$, then [Li, §2.1] shows that while $f(x)$ and $f(y)$ may be generators of a parametrization with $s^{\prime \prime}=n^{\prime \prime}$, the parametrization with $\tilde{x}=f(x), \tilde{y}=f(x)^{-m^{\prime \prime}-t^{\prime \prime}-1} f(y)$ allows us to set $s^{\prime \prime}=n^{\prime \prime}-1$, as required. If $s^{\prime \prime}=n$, then $s^{\prime \prime}=n^{\prime \prime}$; otherwise, $s^{\prime \prime}=s^{\prime}<n$, but then we must have that $s^{\prime \prime}=s^{\prime}=n^{\prime}-1=n^{\prime \prime}-1$.

For all three cases, then, we have that $\left(c^{\prime \prime}, n^{\prime \prime}, m^{\prime \prime}, t^{\prime \prime}, s^{\prime \prime}\right)$ is one of the set of presentations of section 6.1, from [Li, §2.1]. By Proposition 5.1 the presentations 
of $G$ are unique, given a fixed $\langle x\rangle$; we then have that $n=n^{\prime \prime}, m=m^{\prime \prime}, t=t^{\prime \prime}$, and $s=s^{\prime \prime}$. Hence the set of $H$ which admit a surjection of the type described to $G$ must have certain parameters fixed: in cases 1 and $2, m^{\prime}=m$ and $t^{\prime}=t$, while in case 3 , we have only that $m^{\prime}=m$. The remaining parameters may then vary. In case 1 , since $t^{\prime} \in\left\{\max \left(0, n^{\prime}-m^{\prime}-1\right), n^{\prime}-1\right\}$, we must have that $n^{\prime}-m-1 \leq t$ or $n^{\prime} \leq t+m+1$. If $s=n \leq m$, then $n \leq s^{\prime} \leq \min \left(m, n^{\prime}\right)$, and the range of such $s^{\prime}$ is nonempty for any $n^{\prime} \leq t+m+1$. If $s \neq n$, we have that $n^{\prime}-t^{\prime}-1 \leq s^{\prime}$ or that $n^{\prime} \leq s+t+1$, so that $n^{\prime} \leq \min (t+m+1, s+t+1)$. Case 2 is similar: since $t^{\prime} \in\left\{\max \left(0, n^{\prime}-m^{\prime}-2\right), n^{\prime}-2\right\}$, we must have that $n^{\prime}-m-2 \leq t$ or $n^{\prime} \leq t+m+2$. If $s=n \leq m$, then $n \leq s^{\prime} \leq \min \left(m, n^{\prime}\right)$, and the range of such $s^{\prime}$ is nonempty for any $n^{\prime} \leq t+m+2$. If $s \neq n$, we have that $n^{\prime}-t^{\prime}-2 \leq s^{\prime}$ or that $n^{\prime} \leq s+t+2$, so that $n^{\prime} \leq \min (t+m+2, s+t+2)$.

For case 3 , we have more choices, as follows. We have that $t=\min \left(t^{\prime}, n-2\right)$ and $s=\min \left(s^{\prime}, n\right)$. Now if $t<n-2$, then $t^{\prime}=t$ and then $n^{\prime}-m^{\prime}-2 \leq t^{\prime}=t$ and hence $n^{\prime} \leq t+m+2$. If $t=n-2$, however, $t^{\prime}$ may vary anywhere in $\left\{\max \left(n-2, n^{\prime}-m-2\right), \cdots, n^{\prime}-2\right\}$. If $s=n$ or $s=m+t+1=n-1$, then $s^{\prime}$ may vary to take any value in $\left\{n^{\prime}-1, \min \left(n^{\prime}, m+t^{\prime}+1\right)\right\}$, otherwise no surjection exists with $n^{\prime}>n$.

\section{REFERENCES}

[Bl] E. Black, Deformation of dihedral 2-group extensions of fields, Trans. Amer. Math. Soc. 351 (1999), 3229-3241. MR 99m:12004

[CHR] S. Chase, D. Harrison, and A. Rosenberg, Galois theory and Galois cohomology of commutative rings, Mem. Amer. Math. Soc., vol. 52, American Mathematical Society, Providence, RI, 1965; reprinted with corrections, 1968. MR 33:4118

[Cr] T. Crespo, Galois representations, embedding problems and modular forms, Journées Arithmétiques (Barcelona, 1995), Collect. Math. 48 (1997), 63-83. MR 98j:11101

[DI] F. DeMeyer and E. Ingraham, Separable algebras over commutative rings, Lecture Notes in Mathematics, vol. 181, Springer-Verlag, Berlin, 1971. MR 43:6199

[GSS] H. Grundman, T. Smith, and J. Swallow, Groups of order 16 as Galois groups, Exposition. Math. 13 (1995), 289-319. MR 96h:12005

[Ho] K. Hoechsmann, Zum Einbettungsproblem, J. Reine Angew. Math. 229 (1968), 81-106. MR 39:5507

[Ik] M. Ikeda, Zur Existenz eigentlicher galoisscher Körper beim Einbettungsproblem für galoissche Algebren, Abh. math. Sem. Hamburg 24 (1960), 126-131. MR 22:12103

[ILF] I. Ishkhanov, B. Lur'e, and D. Faddeev, The embedding problem in Galois theory, Translations of Mathematical Monographs, vol. 165, American Mathematical Society, Providence, RI, 1997. MR 98c:12007

[Le] A. Ledet, Embedding problems with cyclic kernel of order 4, Israel J. Math. 106 (1998), 109-132. MR 99k:12009

[Li] S. Liedahl, Presentations of metacyclic p-groups with applications to K-admissibility questions, J. Algebra 169 (1994), 965-983. MR 96a:20043

[Sc] L. Schneps, On cyclic field extensions of degree 8, Math. Scand. 71 (1992), 24-30. MR 94d:12004

[Sw1] J. Swallow, Embedding problems and the $C_{16} \rightarrow C_{8}$ obstruction, Contemporary Mathematics 186: Recent Developments in the Inverse Galois Problem, American Mathematical Society, Providence, RI, 1995, pp. 75-90. MR 96j:12010

[Sw2] J. Swallow, Solutions to central embedding problems are constructible, J. Algebra 184 (1996), 1041-1051. MR 97e:12007

Department of Mathematics, University of Oklahoma, Norman, Oklahoma 73019

Current address: 131 Salina Street, Lafayette, Colorado 80026

E-mail address: eblack@math.ou.edu

Department of Mathematics, Davidson College, Davidson, North Carolina 28036

E-mail address: joswallow@davidson.edu 